\title{
Relativistic Quantum Billiards with Threefold Rotational Symmetry: Exact, Symmetry-Projected Solutions for Equilateral Neutrino Billiard
}

\begin{abstract}
B. DieTz*
Lanzhou Center for Theoretical Physics and the Gansu Provincial Key Laboratory of Theoretical Physics, Lanzhou University, Lanzhou, Gansu 730000, China

Doi: 10.12693/APhysPolA.140.473

*e-mail: dietz@lzu.edu.cn

We present analytical results for the eigenvalues and eigenfunctions of a relativistic neutrino billiard with the shape of an equilateral triangle, which are valid from the ultrarelativistic to the nonrelativistic limit. The transition is performed by increasing the mass of the neutrino from zero to infinity. Here, we exploit the threefold symmetry of the triangle to separate the eigenstates according to their transformation properties with respect to rotation by $\frac{2 \pi}{3}$ into three subspaces labeled by $l=0,1,2$. Generally, the boundary condition imposed on the spinor eigenfunctions of the Dirac Hamiltonian of a neutrino billiard with threefold symmetry, in order to confine the neutrino to the billiard domain, yields a relation of the first spinor component to the second one, where both belong to distinct irreducible representations. In order to obtain information on the effect of this entanglement of different symmetry classes on the eigenstates, we investigate the spectral properties and properties of the eigenfunctions for different masses and the transition from the ultrarelativistic to the nonrelativistic limit.
\end{abstract}

topics: relativistic quantum billiards, semiclassical theory, discrete symmetries

\section{Introduction}

A classical billiard (CB) [1-3] consists of a bounded two-dimensional domain, in which a point particle moves freely and is reflected specularly at the boundary. Billiards have the particular property that the features of their classical dynamic can be controlled by an appropriate choice of their shape. Therefore, they are commonly employed for theoretical, numerical, and experimental investigations within the field of quantum chaos [4-7]. The eigenstates of the corresponding nonrelativistic quantum billiard (QB) are obtained by imposing on the solutions of the free-space Schrödinger equation the Dirichlet boundary condition (BC) that they vanish along the boundary. Numerous numerical and experimental studies of QBs have been performed [5, 8-13], confirming the BohigasGiannoni-Schmit (BGS) conjecture [14-17] and the Berry-Tabor (BT) conjecture [18]. They state that the spectral properties of typical quantum systems with fully chaotic and integrable classical dynamic are universal and coincide for sufficiently large energies with those of random matrices from the Gaussian ensembles $[7,19]$ and uncorrelated random numbers drawn from a Poisson process, respectively. Criteria for the applicability of random matrix theory could be finally identified [20] in the semiclassical limit $\hbar \rightarrow 0$ within the periodic orbit (PO) theory, which was pioneered by Gutzwiller [20, 21] and provides an approximation for the fluctuating part of the spectral density of a quantum system in terms of a sum over the POs of the associated classical dynamic, like, for example the renown trace formula of Gutzwiller for chaotic systems.

Berry and Mondragon already proposed in 1987 within the field of relativistic quantum chaos neutrino billiards [23] (NBs). They are governed by the Weyl equation [24] for a non-interacting, massless spin- $1 / 2$ particle - referred to as Dirac equation in [23] and, generally, in that field - whose motion is confined to the billiard area by imposing on its solutions the $\mathrm{BC}$ that the outward current vanishes. Note that such billiards were given the name "neutrino billiards" at a time, where it was not known yet that neutrinos have a mass. Our studies will include massive spin- $1 / 2$ particles and we will take over this denotation, because we use the same BCs as in [23]. In distinction to the nonrelativistic case the associated Dirac Hamiltonian is not invariant under time reversal, implying that the spectral properties of NBs differ from those of the corresponding QB, if their shape generates a chaotic classical dynamic and has no mirror symmetry. Furthermore, for massless neutrinos the length spectrum, i.e., the Fourier transform of the fluctuating part of the spectral density from wave number to length, which for QBs has peaks at the lengths of POs of the associated $\mathrm{CB}$, does not exhibit peaks at the lengths of POs with an odd 
number of reflections at the boundary [25, 26]. Interest in NBs re-emerged with the pioneering fabrication of graphene [27]. The reason is that its band structure exhibits a linear dispersion relation near the Fermi energy, implying that the electronic properties are effectively described by a Dirac equation for spin- $1 / 2$ particles and leading to the relativistic features of graphene [27-29]. This triggered an immense amount of experimental and theoretical studies of finite-size graphene sheets, referred to as graphene billiards [26, 30-41]. In [42] the results of [23] were extended to neutrinos of finite mass $m$ and a relativistic analogue of Gutzwiller's trace formula for QBs with a chaotic dynamic was derived. Furthermore, based on these results the transition from the ultrarelativistic $(m=0)$ to the nonrelativistic limit $(m \rightarrow \infty)$ [43] was considered for a NB with chaotic classical dynamic and the circular billiard; see also [44] where this transition was investigated based on quantum scars. In [41] analytical solutions for the eigenstates and a trace formula were derived for massless elliptic NBs. The extension to massive NBs is straightforward, as will be demonstrated in a forthcoming work.

In the present article we introduce exact analytical, symmetry-projected eigenstates [45-52] for an equilateral-triangle (ET) massive NB. Its shape exhibits a threefold rotational symmetry and mirror symmetries with respect to its three main axes, i.e., it belongs to the $C_{3 v}$ symmetry group. The ET billiard has an integrable dynamic since there exist two constants of motion. To be precise, it exhibits the unusual feature that is has three constants of motion, of which only two are independent [53]. Furthermore, its Hamiltonian is not separable like, e.g., for a rectangular, circular, or elliptic billiard. The quantization of the wave equation in such systems is thus not straightforward. The wave equation for the normal modes and eigenfrequencies of a membrane that is fixed along the sides of an equilateral triangle, which is mathematically identical to the two-dimensional Helmholtz equation with Dirichlet $\mathrm{BCs}$ and the Schrödinger equation for the $\mathrm{QB}$ of corresponding shape, was first solved by Lamé in 1852. Later on, various other derivations were proposed $[54,55]$. The eigenstates of a dielectric ET billiard have been computed numerically in $[56,57]$ on the basis of a superscar model. Due to the unusual features the spectral properties do not comply with those of random Poissonian numbers, i.e., the ET is not a typical integrable system. Naturally, the question arises whether these unusual features of the ET also have an effect on the spectral properties of the ET NB. Note that those of the elliptic and circular massless and massive NBs comply with the BT conjecture [41, 42, 44].

In the present article we investigate the spectral properties of massive ET NBs from the ultrarelativistic to the nonrelativistic limit. For ET GBs analytical results have been presented in [35, 58-60]. In [61] analytical solutions were derived for massless spin-1/2 particles which were confined to the triangular domain by imposing the Dirichlet boundary condition on one component of the spinor wave functions. The differing boundary conditions of this relativistic quantum billiard and the ET NB result in dissimilar eigenstates and features, the most crucial discrepancy being the entanglement of the symmetry classes of the two wave function components for the NB through its BCs, as outlined in the present article. We will briefly review in Sect. 2 the Dirac equation of massive NBs and the associated BCs and the effect of mirror symmetries and discrete rotational symmetries on its eigenstates. Actually, as outlined in $[62,63]$ and in Sect. 2, the eigenstates do not exhibit reflection symmetries with respect to the symmetry axes of the ET. Thus they can only be classified according to their transformation properties with respect to rotations by $\frac{2 \pi}{3}$ into three symmetry classes labelled by $l=0,1,2$. We reveal that the symmetry-projected solutions of the first component characterized by $l$ are linked to the second component belonging to the symmetry group $\tilde{l}=l-1$ with $\tilde{l}=-1 \equiv \tilde{l}=2[64]$. The aim of these studies is to understand the implications of this entanglement of symmetry classes on the properties of the eigenstates. In Sect. 3 the derivation of the exact analytic solutions of massive $\mathrm{NBs}$ is outlined and results are presented for the spectral properties, wave functions and currents in the ultrarelativistic and nonrelativistic limits and for finite, nonzero mass.

\section{The Dirac equation for NBs and the effect of symmetries}

The Dirac equation for a free spin- $1 / 2$ particle with mass $m$ moving with momentum $\hat{\boldsymbol{p}}=-\mathrm{i} \hbar \nabla$ in the two-dimensional $\boldsymbol{r}=(x, y)$ plane is given by

$$
\begin{aligned}
& \hat{H}_{\mathrm{D}} \boldsymbol{\psi}=\left(c \hat{\boldsymbol{\sigma}} \cdot \hat{\boldsymbol{p}}+m c^{2} \hat{\boldsymbol{\sigma}}_{\boldsymbol{z}}\right) \boldsymbol{\psi}=E \boldsymbol{\psi}, \\
& \boldsymbol{\psi}=\left(\begin{array}{c}
\psi_{1} \\
\psi_{2}
\end{array}\right),
\end{aligned}
$$

with $\hat{H}_{\mathrm{D}}$ denoting the Dirac Hamiltonian. Here, $\hat{\boldsymbol{\sigma}}=\left(\hat{\boldsymbol{\sigma}}_{x}, \hat{\boldsymbol{\sigma}}_{y}\right), \hat{\boldsymbol{\sigma}}_{x, y, z}$ stands for the Pauli matrices, $E=c \hbar k_{E}=c \hbar k \sqrt{1+\beta^{2}}$ is the energy of the particle, $k$ is the free-space wave vector and $\beta=\frac{m c}{\hbar k}$ is the ratio of the rest-energy momentum and free-space momentum. The particle is confined to the billiard domain by imposing boundary conditions (BCs) on the wave function components $\psi_{1,2}(\boldsymbol{r})$ which solve the Dirac equation (1), yielding a quantization condition of which the solutions are the eigenvalues and eigenfunctions of the associated Hamiltonian $\hat{H}_{N B}$. We chose the BCs for NBs which were introduced in [23] for $m=0$. The boundary $\boldsymbol{r}_{\mathcal{B}}$ of the triangular billiard is parametrized in Cartesian coordinates in terms of the arc-length parameter $s \in[0, \mathcal{L}]$, where $\mathcal{L}$ denotes the perimeter, $\boldsymbol{r}_{\mathcal{B}}=\left[x_{\mathcal{B}}(s), y_{\mathcal{B}}(s)\right]$, or in the complex plane $w_{\mathcal{B}}(s)=x_{\mathcal{B}}(s)+\mathrm{i} y_{\mathcal{B}}(s)$. The BCs are deduced from the requirement that 
the Hamiltonian of a closed system must have real eigenvalues, that is, it must be self-adjoint, i.e., Hermitian implying that the outgoing current must vanish [23]. Since hermiticity is not destroyed by the additional mass term, the $\mathrm{BC}$ is independent of mass and

$$
\psi_{2}(s) \stackrel{!}{=} \mathrm{i} \mathrm{e}^{\mathrm{i} \alpha(s)} \psi_{1}(s)
$$

with $\alpha(s)$ denoting the angle of the outwardpointing normal vector at $s$ with respect to the $x$-axis. Here, the symbol $\stackrel{!}{=}$ is employed for an equality imposed, e.g., by the BC.

The $\mathrm{BC}$ imposes a phase relation on the wavefunction components $\psi_{1,2}(s)$ at $\boldsymbol{r}_{\mathcal{B}}$. Introducing local coordinates $(n, s)$ along the boundary $(n=0, s)$ in the directions of the normal vector $\boldsymbol{n}(s)=$ $[\cos (\alpha(s)), \sin (\alpha(s))]$ and of the tangential vector $\boldsymbol{t}(s)=[-\sin (\alpha(s)), \cos (\alpha(s))]$ to $\boldsymbol{r}_{\mathcal{B}}(s)$ and writing $\partial_{x} \pm \mathrm{i} \partial_{y}=\mathrm{e}^{ \pm \mathrm{i} \alpha(s)}\left(\partial_{n} \pm \mathrm{i} \partial_{s}\right)$ along the boundary in terms of the normal and tangential derivatives $\partial_{n}=\boldsymbol{n} \cdot \nabla$ and $\partial_{s}=\boldsymbol{t} \cdot \nabla$, respectively, the $\mathrm{BC}$ can be turned into separate ones for each component, i.e.,

$$
\begin{aligned}
& \left.\left(\partial_{n}+\mathrm{i} \partial_{s}\right) \psi_{1}(n, s)\right|_{n \rightarrow 0^{-}} \stackrel{!}{=}-k \mathcal{K}^{-1} \psi_{1}(s), \\
& \left.\left(\partial_{n}-\mathrm{i} \partial_{s}\right) \psi_{2}(n, s)\right|_{n \rightarrow 0^{-}} \stackrel{!}{=} k \mathcal{K} \psi_{2}(s) .
\end{aligned}
$$

Here, $n \rightarrow 0^{-}$means that the boundary is approached in direction of $\boldsymbol{n}(s)$ from the interior and $\psi_{1,2}(n=0, s)=\psi_{1,2}(s)$. Furthermore,

$$
\mathcal{K}=\sqrt{\frac{1-\sin \left(\theta_{\beta}\right)}{1+\sin \left(\theta_{\beta}\right)}},
$$

where

$$
\begin{aligned}
& \cos \left(\theta_{\beta}\right)=\frac{1}{\sqrt{1+\beta^{2}}}, \\
& \sin \left(\theta_{\beta}\right)=\frac{\beta}{\sqrt{1+\beta^{2}}} .
\end{aligned}
$$

The nonrelativistic limit $E \simeq m c^{2}$, is reached for $\beta=\frac{m c}{\hbar k} \rightarrow \infty$ for fixed, nonzero $\hbar k$ or, equivalently for a given range of $k$ values for $\tilde{\beta}=\frac{m c}{\hbar} \gg k$ [43], corresponding to $\mathcal{K} \simeq \frac{1}{2 \beta} \rightarrow 0$ and $\theta_{\beta} \rightarrow \pi / 2$. It complies with the BCs for massive NBs yielding that the spinor components decouple and their wave equations coincide with those of QBs subject to the Robin BCs for sufficiently large $\tilde{\beta}$, which eventually turn into the Dirichlet BCs with increasing $\tilde{\beta}$ [42]. Introducing a two-component spinor $\tilde{\boldsymbol{\psi}}(\boldsymbol{r})$ which is obtained by dividing the components $\psi_{1,2}(\boldsymbol{r})$ in (1) by the amplitudes of the corresponding free-space wave-function components [43]

$$
\boldsymbol{\psi}(\boldsymbol{r})=\sqrt{\frac{1}{2}}\left(\begin{array}{l}
\sqrt{1+\sin \left(\theta_{\beta}\right)} \tilde{\psi}_{1}(\boldsymbol{r}) \\
\sqrt{1-\sin \left(\theta_{\beta}\right)} \tilde{\psi}_{2}(\boldsymbol{r})
\end{array}\right)
$$

turns (1) into a Dirac equation for massive NBs

$$
\begin{aligned}
& \qquad \tilde{\boldsymbol{\psi}}(\boldsymbol{r})+\mathrm{i} \hat{\boldsymbol{\sigma}} \cdot \nabla \tilde{\boldsymbol{\psi}}(\boldsymbol{r})=0 \\
& \text { and } \\
& \tilde{\psi}_{2}(s) \stackrel{!}{=} \mathrm{i}^{\mathrm{i} \alpha(s)} \mathcal{K}^{-1} \tilde{\psi}_{1}(s),
\end{aligned}
$$

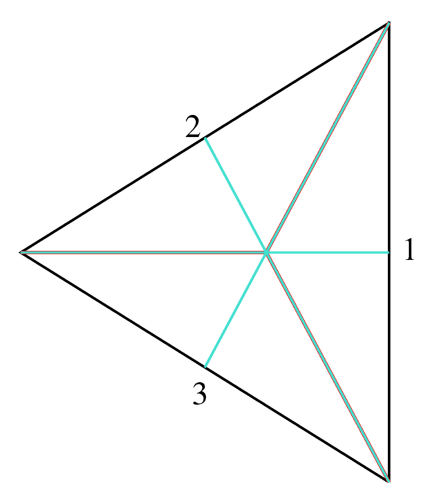

Fig. 1. Fundamental domains of the ET. The rotationally invariant wave functions associated with the irreducible representation $l=0$ obey either the Dirichlet or Neumann conditions at all three symmetry axes (thin cyan lines) and the fundamental domain is $1 / 6$ of the triangle bounded by a fat redcyan line, a thin cyan line and the outer boundary (black line), i.e., one half of a fundamental domain associated with the irreducible representations of the $C_{3}$ symmetry group. Wave functions associated with $l=1,2$ obey either the Dirichlet or Neumann BCs along one of the symmetry axes and the fundamental domain is one half of the triangle.

which has the same form as for the massless NB (1) except that the $\mathrm{BC}$ is modified, and approaches it for $\tilde{\beta} \rightarrow 0$. In the nonrelativistic limit the second wave function component becomes vanishingly small as $\sin \left(\theta_{\beta}\right) \rightarrow 0$.

The ET exhibits mirror-symmetries and a threefold rotational symmetry. Consequently, the eigenstates of the corresponding $\mathrm{QB}$ can be separated into six symmetry classes, namely two with nondegenerate eigenvalues and eigenfunctions which are either symmetric or antisymmetric with respect to all three symmetry axes, and two with degenerate ones and eigenfunctions that are either symmetric or antisymmetric with respect to one of the symmetry axes. Accordingly, the former fulfill the Neumann, respectively, the Dirichlet BCs along all axes and the fundamental domain is $1 / 6$ of the ET bounded by a thin cyan line, the fat red-cyan line and the outer boundary of the ET in Fig. 1. The latter one fulfills either of the BCs along the axis of mirror-symmetry and its fundamental domain is one half of the ET. The eigenfunctions of mirrorsymmetric NBs do not exhibit a reflection symmetry and can only be classified according to their transformation properties under rotation by $\frac{2 \pi}{3}$, as outlined in the following.

Generally, when applying an orthogonal transformation to $\boldsymbol{r}^{\prime}=\hat{R} \cdot \boldsymbol{r}$, or equivalently, a unitary transformation $\hat{U}$ to the Dirac Hamiltonian $\hat{H}_{\mathrm{D}}(\boldsymbol{r})$ with eigenfunctions $\tilde{\boldsymbol{\psi}}(\boldsymbol{r}) \quad\left(\hat{H}_{\mathrm{D}}\left(\boldsymbol{r}^{\prime}\right)=\hat{H}_{\mathrm{D}}^{\prime}=\hat{U}^{\dagger} \hat{H}_{\mathrm{D}} \hat{U}\right)$, then $\overline{\boldsymbol{\psi}}\left(\boldsymbol{r}^{\prime}\right)=\hat{U}^{\dagger} \tilde{\boldsymbol{\psi}}(\boldsymbol{r})$ are the eigenfunctions of $\hat{H}_{\mathrm{D}}^{\prime}$. The unitary operators corresponding to a mirror reflection $\hat{R}_{x, y}= \pm \hat{\sigma}_{z}$ at the $x$ - or the $y$-axis, 
respectively, are $\hat{U}_{x}=\hat{\sigma}_{x}$ or $\hat{U}_{y}=\mathrm{i} \hat{\sigma}_{y}$ yielding for the eigenfunctions $\overline{\boldsymbol{\psi}}_{x}^{\mathrm{T}}\left(\boldsymbol{r}^{\prime}\right)$ of $\hat{H}_{\mathrm{D}}^{\prime}=\hat{H}_{\mathrm{D}}\left(\hat{R}_{x, y} \cdot \boldsymbol{r}\right)$ in terms of the eigenfunction components $\tilde{\psi}_{1}(\boldsymbol{r})$ and $\tilde{\psi}_{2}(\boldsymbol{r})$ of $\hat{H}_{\mathrm{D}}$. These are written as

$$
\overline{\boldsymbol{\psi}}_{x}^{\mathrm{T}}\left(\boldsymbol{r}^{\prime}\right)=\left(\tilde{\psi}_{2}(\boldsymbol{r}), \tilde{\psi}_{1}(\boldsymbol{r})\right)
$$

and

$$
\overline{\boldsymbol{\psi}}_{y}^{\mathrm{T}}\left(\boldsymbol{r}^{\prime}\right)=\left(-\tilde{\psi}_{2}(\boldsymbol{r}), \tilde{\psi}_{1}(\boldsymbol{r})\right) .
$$

However, because of the transformation properties of the normal vector of a NB with mirror symmetry along the $y$ or $x$ axis, $\mathrm{e}^{\mathrm{i} \alpha(-x, y)}=\mathrm{e}^{-\mathrm{i} \alpha(x, y)}$, respectively, $\mathrm{e}^{\mathrm{i} \alpha(x,-y)}=-\mathrm{e}^{-\mathrm{i} \alpha(x, y)}$, the fact that $\boldsymbol{\psi}(\boldsymbol{r})$ is an eigenfunction of that NB does not imply that $\tilde{\boldsymbol{\psi}}\left(\boldsymbol{r}^{\prime}\right)$ fulfills the BC $\bar{\psi}_{2}(s)=\mathrm{i} \mathrm{e}^{\mathrm{i} \bar{\alpha}(s)} \mathcal{K}^{-1} \bar{\psi}_{1}(s)[62]$. Consequently, the eigenfunctions of NBs with just one mirror symmetry are neither symmetric nor antisymmetric under reflection with respect to the symmetry axis.

A coordinate transformation corresponding to a counterclockwise rotation by $\frac{2 \pi}{N}, \boldsymbol{r}^{\prime}=\hat{R}_{N} \cdot \boldsymbol{r}$ with

$$
\hat{R}_{N}=\left(\begin{array}{cc}
\cos \left(\frac{2 \pi}{N}\right) & -\sin \left(\frac{2 \pi}{N}\right) \\
\sin \left(\frac{2 \pi}{N}\right) & \cos \left(\frac{2 \pi}{N}\right)
\end{array}\right),
$$

is performed by applying to $\hat{H}_{\mathrm{D}}$ the unitary operator

$$
\hat{\boldsymbol{U}}_{N}=\left(\begin{array}{cc}
\mathrm{e}^{\mathrm{i} \pi / N} & 0 \\
0 & \mathrm{e}^{-\mathrm{i} \pi / N}
\end{array}\right) .
$$

The eigenfunctions of the transformed Hamiltonian are obtained from those of $\hat{H}_{\mathrm{D}}$ as

$$
\overline{\boldsymbol{\psi}}\left(\boldsymbol{r}^{\prime}\right)=\left(\begin{array}{c}
\bar{\psi}_{1}\left(\boldsymbol{r}^{\prime}\right) \\
\bar{\psi}_{2}\left(\boldsymbol{r}^{\prime}\right)
\end{array}\right)=\left(\begin{array}{c}
\mathrm{e}^{-\mathrm{i} \pi / N} \tilde{\psi}_{1}(\boldsymbol{r}) \\
\mathrm{e}^{\mathrm{i} \pi / N} \tilde{\psi}_{2}(\boldsymbol{r})
\end{array}\right) .
$$

Accordingly, if the billiard shape has an $N$-fold rotational symmetry $w\left(s^{\prime}\right)=\mathrm{e}^{\mathrm{i} \frac{2 \pi}{N}} w(s)$ and $\mathrm{e}^{\mathrm{i} \alpha\left(s^{\prime}\right)}=$ $\mathrm{e}^{\mathrm{i} \frac{2 \pi}{N}} \mathrm{e}^{\mathrm{i} \alpha(s)}$, then the boundary condition for $\bar{\psi}_{1,2}(\boldsymbol{r})$ becomes

$$
\mathrm{e}^{\mathrm{i} \frac{\pi}{N}} \tilde{\psi}_{2}(s) \stackrel{!}{=} \mathrm{i} \mathrm{e}^{\mathrm{i} \frac{2 \pi}{N}} \mathrm{e}^{\mathrm{i} \alpha(s)} \mathcal{K}^{-1} \mathrm{e}^{-\mathrm{i} \frac{\pi}{N}} \tilde{\psi}_{1}(s),
$$

which is fulfilled for $\overline{\boldsymbol{\psi}}\left(\boldsymbol{r}^{\prime}\right)$ if $\tilde{\boldsymbol{\psi}}(\boldsymbol{r})$ is an eigenfunction of $\hat{H}_{N B}$.

The symmetry group is given by $\boldsymbol{G}=C_{N}=$ $\left\{\boldsymbol{e}, \boldsymbol{g}, \ldots, \boldsymbol{g}^{\boldsymbol{N - 1}}\right\}$, where $\boldsymbol{e}$ is the identity operation and the group element $\boldsymbol{g}$ stands for rotation by $\frac{2 \pi}{N}$. The Hamiltonian $\hat{H}_{N B}(\boldsymbol{r})$ can be brought to a block diagonal form according to the $N$ onedimensional irreducible representations labeled by $l=0, \ldots, N-1$ and given by $\mathcal{M}^{(l)}\left(\boldsymbol{g}^{\lambda}\right)=\mathrm{e}^{\mathrm{i} 2 \lambda l / N}$ with $\lambda=0, \ldots N-1$. It means that its eigenstates can be grouped into $N$ subspaces defined by their transformation properties under a rotation by $\frac{2 \pi}{N}$, i.e., when applying the rotation operator $[49,52,65,66]$

$$
\hat{\mathcal{U}}(\boldsymbol{g})=\mathrm{e}^{\mathrm{i} \frac{2 \pi}{N} \hat{L}}
$$

with $\hat{L}$ denoting the angular momentum operator,

$$
\begin{aligned}
& \hat{\mathcal{U}}\left(\boldsymbol{g}_{l}^{\lambda}\right) \tilde{\psi}_{1,2}^{(l)}(\boldsymbol{r})=\psi_{1,2}^{(l)}\left(\boldsymbol{g}_{l}^{-\lambda} \boldsymbol{r}\right)=\tilde{\psi}_{1,2}^{(l)}\left(\boldsymbol{r}^{\prime}\right)= \\
& \quad \mathrm{e}^{\mathrm{i} \lambda \frac{2 \pi}{N} l} \tilde{\psi}_{1,2}^{(l)}(\boldsymbol{r}) .
\end{aligned}
$$

Note that the Dirac equation (10), and the $\mathrm{BC}$ (11) relate components of the wave function with different transformation properties under rotation by $\frac{2 \pi}{N}$ [64]. Assuming that

$$
\tilde{\psi}_{1}^{(l)}\left(\boldsymbol{r}^{\prime}\right)=\mathrm{e}^{-\mathrm{i} \frac{2 \pi}{N} l} \tilde{\psi}_{1}^{(l)}(\boldsymbol{r}),
$$

where $\boldsymbol{r}^{\prime}=\hat{R}_{N} \boldsymbol{r}$, and replacing accordingly the first component on both sides of

$$
\begin{aligned}
& \hat{H}_{\mathrm{D}}\left(\boldsymbol{r}^{\prime}\right) \overline{\boldsymbol{\psi}}\left(\boldsymbol{r}^{\prime}\right)= \\
& \left(\begin{array}{cc}
0 & \frac{\partial}{\partial x^{\prime}}-\mathrm{i} \frac{\partial}{\partial y^{\prime}} \\
\frac{\partial}{\partial x^{\prime}}+\mathrm{i} \frac{\partial}{\partial y^{\prime}} & 0
\end{array}\right)\left(\begin{array}{c}
\mathrm{e}^{-\mathrm{i} \frac{\pi}{N}} \tilde{\psi}_{1}(\boldsymbol{r}) \\
\mathrm{e}^{\mathrm{i} \frac{\pi}{N}} \tilde{\psi}_{2}(\boldsymbol{r})
\end{array}\right)= \\
& \mathrm{i} k\left(\begin{array}{c}
\mathrm{e}^{-\mathrm{i} \frac{\pi}{N}} \tilde{\psi}_{1}(\boldsymbol{r}) \\
\mathrm{e}^{\mathrm{i} \frac{\pi}{N}} \tilde{\psi}_{2}(\boldsymbol{r})
\end{array}\right)
\end{aligned}
$$

reveals that $\boldsymbol{\psi}\left(\boldsymbol{r}^{\prime}\right)$ solves the Dirac equation if the second component transforms as

$$
\tilde{\psi}_{2}\left(\boldsymbol{r}^{\prime}\right)=\mathrm{e}^{-\mathrm{i} \frac{2 \pi}{N}(l-1)} \tilde{\psi}_{2}(\boldsymbol{r}) \equiv \tilde{\psi}_{2}^{(l-1)}\left(\boldsymbol{r}^{\prime}\right) .
$$

Similarly, using the $N$-fold symmetry and inserting (20) into the BC (11) implies that

$$
\begin{gathered}
\tilde{\psi}_{2}\left(s^{\prime}\right) \stackrel{!}{=} \mathrm{i} \mathrm{e}^{\mathrm{i} \alpha\left(s^{\prime}\right)} \mathcal{K}^{-1} \tilde{\psi}_{1}^{(l)}\left(s^{\prime}\right)= \\
\mathrm{i} \mathrm{e}^{\mathrm{i}\left(\alpha(s)+\frac{2 \pi}{N}\right)} \mathcal{K}^{-1} \mathrm{e}^{-\mathrm{i} \frac{2 \pi}{N} l} \tilde{\psi}_{1}^{(l)}(s)= \\
\mathrm{e}^{-\mathrm{i} \frac{2 \pi}{N}(l-1)} \tilde{\psi}_{2}(s) \equiv \tilde{\psi}_{2}^{(l-1)}\left(s^{\prime}\right) .
\end{gathered}
$$

Hence, the Dirac equation of NBs with an $N$-fold reflection symmetry relates the first component with symmetry class $l$ to the second component with symmetry class $\tilde{l}=(l-1)^{\dagger 1}$. This feature has its origin in the presence of the additional spin degree of freedom. Throughout the article we will specify the irreducible representation of the first wave-function component, i.e., give the value of $l$ for $\tilde{\psi}_{1}^{(l)}(s)$.

\section{Exact solutions for NBs with the shape of an ET}

The shape and fundamental domains associated with the irreducible representations of the $C_{3}$ symmetry group of the ET billiard of side lengths unity and height $h=\frac{\sqrt{3}}{2}$ are shown in Fig. 1 with the boundary of the subdomain marked by 1 given in Cartesian coordinates

$$
\boldsymbol{r}_{0}=\left(\begin{array}{c}
\frac{1}{2 \sqrt{3}} \\
\frac{y}{2}
\end{array}\right), \quad \boldsymbol{r}_{j}=\hat{R}^{j} \boldsymbol{r}_{0},
$$

or in the complex plane as

$$
\begin{aligned}
& w_{0}(s)=\frac{1}{2 \sqrt{3}}+\frac{\mathrm{i}}{2} y, \\
& w_{j}(s)=\mathrm{e}^{\mathrm{i} \frac{2 \pi}{3} j} w_{0}(s),
\end{aligned}
$$

\footnotetext{
$\dagger 1$ Note that a twofold symmetry is equivalent to a mirror symmetry with respect to two perpendicular axes like, e.g., exhibited by the ellipse billiard [41]. Thus the wave function components $\tilde{\psi}_{1,2}(\boldsymbol{r})$ maybe classified according to their transformation properties under rotation by $\pi$, and $\tilde{\psi}_{2}(\boldsymbol{r})$ is antisymmetric if $\tilde{\psi}_{1}(\boldsymbol{r})$ is symmetric under that transformation or vice versa.
} 
with $y \in[-1,1]$ and $\hat{R} \equiv \hat{R}_{3}$ is defined in (14). The boundaries of subdomains 2 and 3 are obtained by rotating $\boldsymbol{r}_{0}$ by $\frac{2 \pi}{3} j$, where $j=1,2$. The ET has a threefold rotational symmetry and mirror symmetries with respect to the three main axes, i.e., $C_{3 v}$ symmetry. Accordingly, the eigenstates of the corresponding $\mathrm{QB}$ can be divided into six fundamental domains with either the Dirichlet or the Neumann BCs along the three symmetry axes [67, 68] or two fundamental domains corresponding to reflection symmetry with respect to one of the symmetry axes. Eigenfunctions that obey the Dirichlet BCs along one of them coincide with those of the right-angled triangular QB obtained by halving the ET along that axis [54].

As outlined in the previous section, the eigenfunctions of the ET NB can be classified according to their transformation properties under rotation by $\frac{2 \pi}{3}$. We write an ansatz for the solutions of the Dirac equation in terms of a superposition of plane waves [62]. For this, we exploit the threefold symmetry and start with an ansatz corresponding to a reflection at the outer boundary of the subdomain 1 in Fig. 1, restricting $\boldsymbol{r}$ to that subdomain. Thus,

$$
\psi(\boldsymbol{r})=a_{0}(k) \mathrm{e}^{\mathrm{i} \boldsymbol{k}_{0} \boldsymbol{r}}+b_{0}(k) \mathrm{e}^{-\mathrm{i} \boldsymbol{k}_{0}^{*} \boldsymbol{r}}
$$

where

$$
\begin{aligned}
& \boldsymbol{k}_{0} \equiv \boldsymbol{k}=\left(k_{x}, k_{y}\right)=k\left(\begin{array}{c}
\cos \left(\theta_{k}\right) \\
\sin \left(\theta_{k}\right)
\end{array}\right), \\
& \boldsymbol{k}_{0}^{*}=\left(k_{x},-k_{y}\right) .
\end{aligned}
$$

Rotating this ansatz twice by $\frac{2 \pi}{3}$ leads to a planewave ansatz for the ET

$$
\tilde{\psi}_{1}(\boldsymbol{r})=\sum_{j=0}^{2}\left[a_{j}(k) \mathrm{e}^{\mathrm{i} \boldsymbol{k}_{j} \boldsymbol{r}}+b_{j}(k) \mathrm{e}^{-\mathrm{i} \boldsymbol{\kappa}_{j} \boldsymbol{r}}\right],
$$

where

$$
\begin{aligned}
& \boldsymbol{k}_{j}=\left(\hat{R}^{\dagger}\right)^{j} \boldsymbol{k}_{0}=k\left(\begin{array}{c}
\cos \left(\theta_{k}-\frac{2 \pi}{3} j\right) \\
\sin \left(\theta_{k}-\frac{2 \pi}{3} j\right)
\end{array}\right), \\
& \boldsymbol{\kappa}_{j}=\left(\hat{R}^{\dagger}\right)^{j} \boldsymbol{k}_{0}^{*}=k\left(\begin{array}{c}
\cos \left(\theta_{k}+\frac{2 \pi}{3} j\right) \\
-\sin \left(\theta_{k}+\frac{2 \pi}{3} j\right)
\end{array}\right),
\end{aligned}
$$

yielding that $\boldsymbol{\kappa}_{1}=\boldsymbol{k}_{2}^{*}$ and $\boldsymbol{\kappa}_{2}=\boldsymbol{k}_{1}^{*}$. Here, we apply

$$
\boldsymbol{k}_{0} \boldsymbol{r}_{j}=\boldsymbol{k} \hat{R}^{j} \boldsymbol{r}=\left(\hat{R}^{\dagger}\right)^{j} \boldsymbol{k} \boldsymbol{r}=\boldsymbol{k}_{j} \boldsymbol{r} .
$$

To construct an ansatz for each of the three subspaces we first consider solutions that are invariant under counterclockwise rotation by $\frac{2 \pi}{3}$, i.e., the set of eigenfunctions with the property

$$
\begin{gathered}
\tilde{\psi}_{1}^{(0)}(\hat{R} \boldsymbol{r})=\sum_{j=0}^{2}\left[a_{j}^{(0)}(k) \mathrm{e}^{\mathrm{i} \boldsymbol{k}_{j} \hat{R} \boldsymbol{r}}+b_{j}^{(0)}(k) \mathrm{e}^{-\mathrm{i} \boldsymbol{\kappa}_{j} \hat{R} \cdot \boldsymbol{r}}\right]= \\
\sum_{j=0}^{2}\left[a_{j}^{(0)}(k) \mathrm{e}^{\mathrm{i} \hat{R}^{\dagger} \boldsymbol{k}_{j} \boldsymbol{r}}+b_{j}^{(0)}(k) \mathrm{e}^{-\mathrm{i} \hat{R}^{\dagger} \boldsymbol{\kappa}_{j} \boldsymbol{r}}\right]= \\
\sum_{j=0}^{2}\left[a_{j}^{(0)}(k) \mathrm{e}^{\mathrm{i} \boldsymbol{k}_{j+1} \boldsymbol{r}}+b_{j}^{(0)}(k) \mathrm{e}^{-\mathrm{i} \boldsymbol{\kappa}_{j+1} \boldsymbol{r}}\right] \stackrel{!}{=} \tilde{\psi}_{1}^{(0)}(\boldsymbol{r})
\end{gathered}
$$

with $\boldsymbol{k}_{3}=\boldsymbol{k}_{0}$ and $\boldsymbol{\kappa}_{3}=\boldsymbol{\kappa}_{0}$. This equality has to hold for any value of $\boldsymbol{r}$, implying with (28) that $a_{0}^{(0)}(k)=a_{1}^{(0)}(k)=a_{2}^{(0)}(k)=a(k)$ and $b_{0}^{(0)}(k)=$ $b_{1}^{(0)}(k)=b_{2}^{(0)}(k)=b(k)$. For general $l=0,1,2$, the transformation condition reads

$$
\begin{gathered}
\tilde{\psi}_{1}^{(l)}(\hat{R} \boldsymbol{r})=\sum_{j=0}^{2}\left[a_{j}^{(l)}(k) \mathrm{e}^{\mathrm{i} \boldsymbol{k}_{j+1} \boldsymbol{r}}+b_{j}^{(l)}(k) \mathrm{e}^{-\mathrm{i} \boldsymbol{\kappa}_{j+1} \boldsymbol{r}}\right] \stackrel{!}{=} \\
\mathrm{e}^{-\mathrm{i} \frac{2 \pi}{3} l} \tilde{\psi}_{1}^{(l)}(\boldsymbol{r})
\end{gathered}
$$

yielding

$$
a_{j}^{(l)}(k)=\mathrm{e}^{-\mathrm{i} \frac{2 \pi}{3} l} a_{j+1}^{(l)}(k) \Rightarrow a_{j}^{(l)}(k)=a(k) \mathrm{e}^{\mathrm{i} \frac{2 \pi}{3} l j}
$$

and

$$
b_{j}^{(l)}(k)=\mathrm{e}^{-\mathrm{i} \frac{2 \pi}{3} l} b_{j+1}^{(l)}(k) \Rightarrow b_{j}^{(l)}(k)=b(k) \mathrm{e}^{\mathrm{i} \frac{2 \pi}{3} l j} .
$$

Hence, a general ansatz for the first component of $\tilde{\psi}(\boldsymbol{r})$ is given by

$$
\tilde{\psi}_{1}^{(l)}(\boldsymbol{r})=a(k) \sum_{j=0}^{2} \mathrm{e}^{\mathrm{i} \frac{2 \pi}{3} l j}\left[\mathrm{e}^{\mathrm{i} \boldsymbol{k}_{j} \boldsymbol{r}}+c(k) \mathrm{e}^{-\mathrm{i} \boldsymbol{\kappa}_{j} \boldsymbol{r}}\right] .
$$

An ansatz for the second component of $\tilde{\boldsymbol{\psi}}(\boldsymbol{r})$ is obtained by inserting (36) into the Dirac equation (10),

$$
\begin{aligned}
& \tilde{\psi}_{2}^{(l)}(\boldsymbol{r})=\mathrm{i} k\left(\frac{\partial}{\partial x}+\mathrm{i} \frac{\partial}{\partial y}\right) \tilde{\psi}_{1}^{(l)}(\boldsymbol{r})= \\
& a(k) \sum_{j=0}^{2} \mathrm{e}^{\mathrm{i} \frac{2 \pi}{3}(l-1) j} \\
& \quad \times\left[\mathrm{e}^{\mathrm{i} \theta_{k}} \mathrm{e}^{\mathrm{i} \boldsymbol{k}_{j} \boldsymbol{r}}-c(k) \mathrm{e}^{-\mathrm{i} \theta_{k}} \mathrm{e}^{-\mathrm{i} \boldsymbol{\kappa}_{j} \boldsymbol{r}}\right],
\end{aligned}
$$

where we employed (29) and (30). A quantization condition is obtained by imposing the BC (11) along the outer boundary of the subdomain 1 in Fig. 1 with $\mathrm{e}^{-\mathrm{i} \alpha(s)}=1$, i.e., $\tilde{\psi}_{1}^{(l)}\left(\boldsymbol{r}_{0}\right) \stackrel{!}{=}$ $-\mathrm{i} \mathcal{K} \mathrm{e}^{-\mathrm{i} \alpha(s)} \tilde{\psi}_{2}^{(l)}\left(\boldsymbol{r}_{0}\right)$ that is

$$
\begin{aligned}
& \sum_{j=0}^{2} \mathrm{e}^{\mathrm{i} \frac{2 \pi}{3} l j} \mathrm{e}^{\mathrm{i} \boldsymbol{k}_{j} \boldsymbol{r}_{0}}\left[1+\mathcal{K} \mathrm{e}^{\mathrm{i}\left(\theta_{k}-\frac{2 \pi}{3} j+\frac{\pi}{2}\right)}\right]= \\
& -c(k) \sum_{j=0}^{2} \mathrm{e}^{\mathrm{i} \frac{2 \pi}{3} l j} \mathrm{e}^{-\mathrm{i} \boldsymbol{\kappa}_{j} \boldsymbol{r}_{0}}\left[1+\mathcal{K} \mathrm{e}^{-\mathrm{i}\left(\theta_{k}+\frac{2 \pi}{3} j+\frac{\pi}{2}\right)}\right] .
\end{aligned}
$$

By construction of the plane-wave ansatz the BC is fulfilled at all sides of the triangle if it is at $\boldsymbol{r}_{0}$. For all values of $y \in[-1,1],(38)$ must be held, yielding

$$
\begin{aligned}
& c(k)=-\mathrm{e}^{\mathrm{i} 2 k_{x, 0} \rho} \mathrm{e}^{\mathrm{i} 2 \Phi_{0}}, \\
& \mathrm{e}^{\mathrm{i} k_{x, 1} \rho} \mathrm{e}^{-\mathrm{i} l \frac{\pi}{3}} \mathrm{e}^{\mathrm{i} \Phi_{1}}=\mathrm{e}^{\mathrm{i} k_{x, 0} \rho} \mathrm{e}^{\mathrm{i} \Phi_{0}} \mathrm{e}^{-\mathrm{i} n_{1} \pi}, \\
& \mathrm{e}^{\mathrm{i} k_{x, 2} \rho} \mathrm{e}^{\mathrm{i} \frac{\pi}{3} l} \mathrm{e}^{\mathrm{i} \Phi_{2}}=\mathrm{e}^{\mathrm{i} k_{x, 0} \rho} \mathrm{e}^{\mathrm{i} \Phi_{0}} \mathrm{e}^{-\mathrm{i} n_{2} \pi},
\end{aligned}
$$


with $\rho=\frac{1}{2 \sqrt{3}}$ and the notations

$$
\begin{gathered}
\mathrm{e}^{\mathrm{i} \Phi_{j}}=\sqrt{\frac{1+\mathcal{K} \mathrm{e}^{\mathrm{i}\left(\theta_{k}-\frac{2 \pi}{3} j+\frac{\pi}{2}\right)}}{1+\mathcal{K} \mathrm{e}^{-\mathrm{i}\left(\theta_{k}-\frac{2 \pi}{3} j+\frac{\pi}{2}\right)}}}= \\
\frac{k+\mathrm{i} \mathcal{K}\left(k_{x, j}+\mathrm{i} k_{y, j}\right)}{\sqrt{k^{2}\left(1+\mathcal{K}^{2}\right)-2 k \mathcal{K} k_{y, j}}},
\end{gathered}
$$

for $j=0,1,2$. Multiplication of (40) with (41) and division of (40) by (41) give with

$$
\begin{aligned}
& k_{x, 1}+k_{x, 2}=-k_{x, 0}=-k_{x}, \\
& \left(k_{x, 1}-k_{x, 2}\right) \rho=\frac{k_{y, 0}}{2}=\frac{k_{y}}{2},
\end{aligned}
$$

the quantization conditions for $k_{x}$ and $k_{y}$. Namely,

$$
\begin{aligned}
& c(k)=-\mathrm{e}^{\frac{\mathrm{i}}{\sqrt{3}} k_{x}} \mathrm{e}^{\mathrm{i} 2 \Phi_{0}}, \\
& \mathrm{e}^{\mathrm{i} \frac{\sqrt{3}}{2} k_{x}}=\mathrm{e}^{\mathrm{i}\left(n_{1}+n_{2}\right) \pi} \mathrm{e}^{\mathrm{i} \Phi_{a}}, \\
& \mathrm{e}^{\frac{\mathrm{i}}{2} k_{y}}=\mathrm{e}^{\mathrm{i} \frac{2 \pi}{3} l} \mathrm{e}^{\mathrm{i}\left(n_{2}-n_{1}\right) \pi} \mathrm{e}^{\mathrm{i} \Phi_{b}},
\end{aligned}
$$

where

$$
\begin{aligned}
& \mathcal{B}_{a} \mathrm{e}^{\mathrm{i} \Phi_{a}}=\mathcal{B}_{a} \mathrm{e}^{\mathrm{i}\left(\Phi_{1}+\Phi_{2}-2 \Phi_{0}\right)}= \\
& 1+\mathcal{K}^{4}-4 \mathcal{K}^{2}-\mathcal{K}\left(1+\mathcal{K}^{2}\right) \sin \left(\theta_{k}\right) \\
& +4 \mathcal{K}^{2} \sin ^{2} \theta_{k}+3 \mathrm{i} \mathcal{K}\left(\mathcal{K}^{2}-1\right) \cos \left(\theta_{k}\right) \\
& \mathcal{B}_{b} \mathrm{e}^{\mathrm{i} \Phi_{b}}=\mathcal{B}_{b} \mathrm{e}^{\mathrm{i}\left(\Phi_{2}-\Phi_{1}\right)}=1-\frac{\mathcal{K}^{2}}{2}+\mathcal{K} \sin \left(\theta_{k}\right) \\
& -\mathrm{i} \sqrt{3}\left(\frac{\mathcal{K}^{2}}{2}+\mathcal{K} \sin \left(\theta_{k}\right)\right)
\end{aligned}
$$

and $0 \leq \Phi_{a, b} \leq \pi$. Here, $\mathcal{B}_{a, b}$ denote the modulus of the respective term on the right hand side of the equation. The eigenvalues are obtained as the solutions of the set of transcendental equations

$$
\begin{aligned}
& \tan \left(\frac{\sqrt{3}}{2} k_{x}-\tilde{n} \pi\right)= \\
& 3 \frac{\mathcal{K}\left(\mathcal{K}^{2}-1\right) \cos \left(\theta_{k}\right)}{1+\mathcal{K}^{4}-4 \mathcal{K}^{2}-\mathcal{K}\left(1+\mathcal{K}^{2}\right) \sin \left(\theta_{k}\right)+4 \mathcal{K}^{2} \sin ^{2} \theta_{k}} \\
& \tan \left(\frac{k_{y}}{2}-\frac{2 \pi}{l}-\tilde{m} \pi\right)=\sqrt{3} \frac{\frac{1}{2} \mathcal{K}^{2}+\mathcal{K} \sin \left(\theta_{k}\right)}{1-\frac{1}{2} \mathcal{K}^{2}+\mathcal{K} \sin \left(\theta_{k}\right)},
\end{aligned}
$$

with $\tilde{n}=n_{1}+n_{2}$ and $\tilde{m}=n_{2}-n_{1}$. Note that $\Phi_{a}\left(\theta_{k}\right)=-\Phi_{a}\left(\pi-\theta_{k}\right)$ and $\Phi_{b}\left(\theta_{k}\right)=\Phi_{b}\left(\pi-\theta_{k}\right)$. Consequently, if $\left(k_{x}, k_{y}\right)$ is a solution of (48) and (49), then also $\left(-k_{x}, k_{y}\right)$ is one. Furthermore, in the nonrelativistic limit $\tilde{\beta} \rightarrow \infty$ we have $\Phi_{a, b} \rightarrow 0$, so that for $l=0\left( \pm k_{x}, \pm k_{y}\right)$ are solutions if $\left(k_{x}, k_{y}\right)$ is one. Employing this property, the proof that the associated wave functions are real for $l=0$ in the nonrelativistic limit is straightforward.

\subsection{Nonrelativistic limit}

We first consider the nonrelativistic case $m \rightarrow \infty$, i.e., $\sin \theta_{\beta}=1$ and $\mathcal{K}=0$. Then, $\Phi_{j}=0, j=0,1,2$ and

$$
\begin{aligned}
& c(k)=-\mathrm{e}^{\frac{\mathrm{i}}{\sqrt{3}} k_{x}}, \\
& \mathrm{e}^{\mathrm{i} \frac{\sqrt{3}}{2} k_{x}}=\mathrm{e}^{\mathrm{i}\left(n_{1}+n_{2}\right) \pi} \\
& \mathrm{e}^{\frac{\mathrm{i}}{2} k_{y}}=\mathrm{e}^{\mathrm{i} \frac{2 \pi}{3} l} \mathrm{e}^{\mathrm{i}\left(n_{2}-n_{1}\right) \pi}
\end{aligned}
$$

thus leading to the quantization conditions

$$
\begin{aligned}
& \frac{\sqrt{3}}{2 \pi} k_{x}=\left(n_{1}+n_{2}\right), \\
& \frac{3}{2 \pi} k_{y}=2 l+3\left(n_{2}-n_{1}\right) .
\end{aligned}
$$

Here, for $l=0$ the cases $n_{1}=0, n_{2}=0,2 n_{1}=n_{2}$ and $2 n_{2}=n_{1}$, for $l=1$ the cases $2 n_{1}=n_{2}-1$ and $2 n_{2}=n_{1}+1$ and for $l=2$ the cases $2 n_{1}=$ $n_{2}+2$ and $2 n_{2}=n_{1}-2$ need to be excluded, because the corresponding wave functions vanish identically. The dynamic of the ET can be described within the Einstein-Brillouin-Keller torus quantization scheme in action-angle variables. It, actually, has three circuits on its two-dimensional torus with any two of them independent, characterized by the actions

$$
\begin{aligned}
& I_{1}=\frac{\sqrt{3}}{4 \pi} k_{x}+\frac{3}{4 \pi} k_{y}, \\
& I_{2}=\frac{\sqrt{3}}{4 \pi} k_{x}-\frac{3}{4 \pi} k_{y}, \\
& I_{3}=\frac{\sqrt{3}}{2 \pi} k_{x} .
\end{aligned}
$$

With (51) these are quantized as

$$
\begin{aligned}
& I_{1}=2 n_{2}-n_{1}+l, \\
& I_{2}=2 n_{1}-n_{2}-l, \\
& I_{3}=n_{1}+n_{2} .
\end{aligned}
$$

Choosing, e.g. the quantization condition $I_{2}=m$, $I_{3}=n$, i.e.,

$$
k_{x}=\frac{2 \pi}{\sqrt{3}} n, \quad k_{y}=\frac{2 \pi}{3}(n-2 m),
$$

yields the eigenvalues $k$

$$
k_{n, m}=\frac{4 \pi}{3} \sqrt{n^{2}+m^{2}-n m}
$$

for $1 \leq m \leq n / 2, n=0,1, \ldots$, where

$$
n_{1}=\frac{m+n+l}{3}, \quad n_{2}=\frac{2 n-m-l}{3} .
$$

The associated wave functions $\psi_{1,2}(\boldsymbol{r})$ are obtained by inserting the quantization results (54) for $k$, $k_{x}, k_{y}$ into (39) to determine $c(k)$ and into (36) and (38), respectively, where $\psi_{2}(\boldsymbol{r})$ approaches zero with increasing $\tilde{\beta}$, i.e., for $\sin \left(\theta_{\beta}\right) \rightarrow 1$, see (6). For $l=0$ the wave functions are invariant under rotation by $\frac{2 \pi}{3}$ and real, whereas for $l=1,2 \hat{R}$ needs to be applied three times on $\psi_{1,2}^{(l)}(\boldsymbol{r})$ in order to recover its initial value and the wave functions are complex, and, thus, not invariant under application of the anti-unitary time-reversal operator $\hat{T}=\hat{K}$ with $\hat{K}$ the complex conjugation operator [7]. The eigenvalues corresponding to $l=1,2$ are identical as expected, because the $\mathrm{QB}$ is time-reversal invariant. 

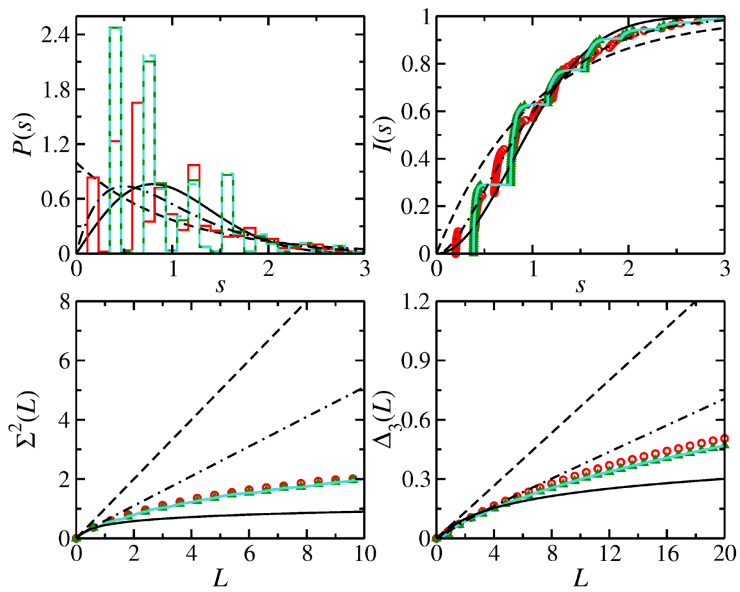

Fig. 2. Spectral properties of the QB. Shown are the results for (a) the nearest-neighbor spacing distribution $P(s)$, (b) the cumulative nearest-neighbor spacing distribution $I(s)$, (c) the number variance $\Sigma^{2}(L)$ and (d) the spectral rigidity $\Delta_{3}(L)$ for the states with $l=0$ (red lines and circles), $l=1$ (dark green lines and triangles) and $l=2$ (dashed turquoise lines). They are compared to the spectral properties of the GOE (black full lines) and Poissonian random numbers (dashed black lines) and semi-Poisson (dash-dotted black lines).

Since the wave functions of the NBs do not exhibit any reflection symmetries with respect to the three symmetry axes of the ET billiard, we did not separate the spectrum for $l=0$ according to the reflection symmetries but, otherwise, took into account degenerate eigenvalues only once. Prior to the analysis of the fluctuation properties in the subspectra of the ET, system specific properties, i.e., the smooth dependence of the spectral density on $k$, needs to be removed. We carried out this unfolding by replacing the eigenvalues $k_{n, m}$ by the smooth part of the integrated spectral density, which is given by Weyl's formula [49] and corresponds to a quadratic polynomial, which depends on the area and the perimeter of the billiard. This yields dimensionless eigenvalues $\epsilon_{i}$ with mean spacing unity. We investigated the spectral properties in terms of the nearest-neighbor spacing distribution $P(s)$, the cumulative nearest-neighbor spacing distribution $I(s)$, the number variance $\Sigma^{2}(L)$ and the Dyson-Mehta statistic $\Delta_{3}(L)$ [19] which gives information on the rigidity of a spectrum. While the former two give information on short-range correlations, the latter two provide a measure for the long-range correlations.

Figure 2 shows the results for $l=0$ (red), $l=1$ (turquoise) and $l=2$ (dark green). The latter lie on top of each other because they are degenerate. For comparison the spectral properties for uncorrelated Poissonian random numbers (black dashed lines), for random matrices from the Gaussian orthogonal ensemble (GOE), which describe

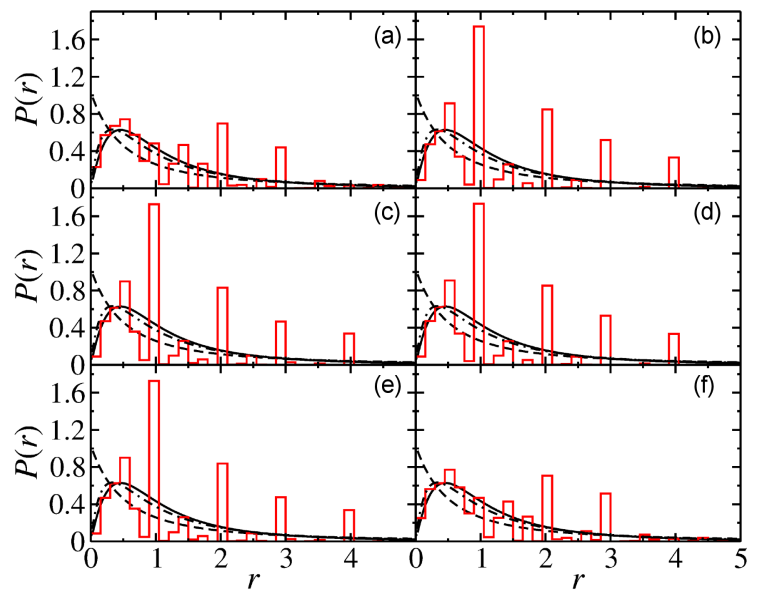

Fig. 3. Ratio distributions of the QB (left) and the NB (right). Shown are the results for the states with $l=0(\mathrm{a}, \mathrm{b}), l=1(\mathrm{c}, \mathrm{d})$ and $l=2(\mathrm{e}, \mathrm{f})$ (red histograms). They are compared to those of the GOE (black solid lines), Poissonian random numbers (dashed black lines) and semi-Poisson (dashdotted black lines).

the spectral properties of typical quantum systems with corresponding chaotic classical dynamic and time-reversal symmetry (black solid lines), are plotted. Furthermore, the spectral properties for semiPoisson statistic [69, 70] are shown (black dashdotted lines), which is encountered in systems that have a statistic intermediate between GOE and Poisson statistic. Interestingly, the nearest-neighbor spacing distribution and its cumulative distribution follow those curves and neither coincide with Poisson nor with GOE statistic. The long-range correlations are also intermediate between Poisson and GOE and well described by the Porter-Rosenzweig model [71] which interpolates between Poisson and GOE. To validate that the discrepancies between the spectral properties and the expected Poisson statistic are not a result of improper unfolding we furthermore considered the distribution of the ratios $r_{i}=\left(\epsilon_{i+1}-\epsilon_{i}\right) /\left(\epsilon_{i}-\epsilon_{i-1}\right)[70,72]$ which is dimensionless so the non-unfolded eigenvalues can be used. The ratio distributions and cumulative ratio distributions shown in the left parts of Figs. 3 and 4 are especially for the cumulative ratio distribution, which does not depend on the binning, surprisingly well described by semi-Poisson statistic [70, 73].

For nonrelativistic quantum systems the direct link between the quantum spectral density and classical periodic orbits is best visualized by length spectra, obtained from the Fourier transform of the spectral density from wave number $k$ to length $l_{k}$, because they exhibit peaks at the lengths of periodic orbits. In Fig. 5 are shown the length spectra for, from bottom to top, the complete eigenvalue spectrum (black) and the subspectra for $l=0$ (red), $l=1$ (dark green) and $l=2$ (violet). The latter two are identical, as expected since the eigenvalue 


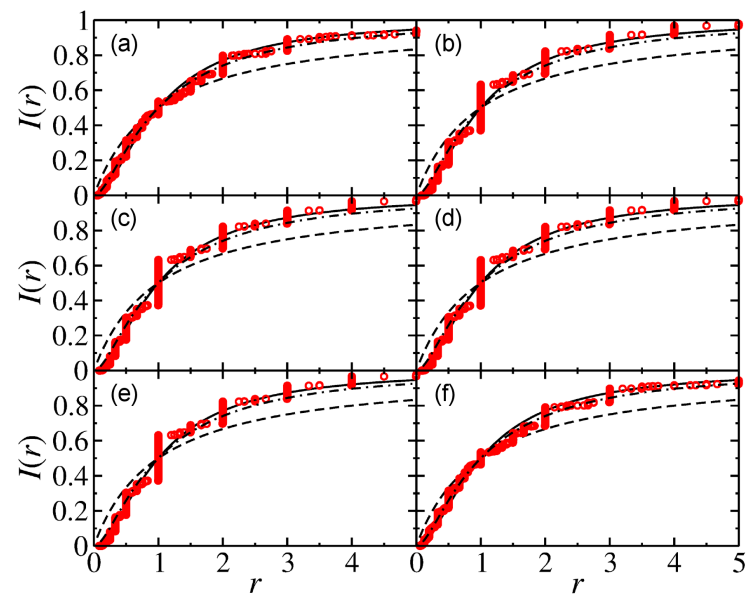

Fig. 4. Description as in Fig. 3, but for the cumulative ratio distributions.

sequences coincide. The symmetry-projected length spectra exhibit peaks at the lengths of the POs of the $\mathrm{CB}$, and additional ones at the lengths of pseudo orbits, which correspond to POs in the respective fundamental domain, but are not periodic when unfolded back to the full system. However, since these pseudo orbits are POs of a fundamental domain, their initial and final points are related in the full system via the symmetry operations of the associated irreducible representation [45, 52]. Most of the peaks corresponding to pseudo orbits are observed in all three symmetry-projected length spectra. This may be attributed to the additional mirror symmetries of the ET.

\subsection{Ultrarelativistic limit}

In the ultrarelativistic limit $m=0$, i.e., $\sin \left(\theta_{\beta}\right)=0$ and $\mathcal{K}=1$, implying that $\Phi_{j}=\theta_{k}+$ $\frac{\pi}{2}-\frac{2 \pi}{3} j, j=0,1,2$, the quantization conditions (45) become

$$
\begin{aligned}
& c(k)=-\mathrm{e}^{\frac{\mathrm{i}}{\sqrt{3}} k_{x}} \mathrm{e}^{\mathrm{i}\left(\theta_{k}+\frac{\pi}{2}\right),} \\
& \mathrm{e}^{\mathrm{i} \frac{\sqrt{3}}{2} k_{x}}=\mathrm{e}^{\mathrm{i}\left(n_{1}+n_{2}\right) \pi}, \\
& \mathrm{e}^{\frac{\mathrm{i}}{2} k_{y}}=\mathrm{e}^{\mathrm{i} \frac{2 \pi}{3}(l+1)} \mathrm{e}^{\mathrm{i}\left(n_{2}-n_{1}\right) \pi},
\end{aligned}
$$

yielding

$$
\begin{aligned}
& \frac{\sqrt{3}}{2 \pi} k_{x}=\left(n_{1}+n_{2}\right) \\
& \frac{3}{2 \pi} k_{y}=2(l+1)+3\left(n_{2}-n_{1}\right) .
\end{aligned}
$$

Here, for $l=0,2 n_{1}=n_{2}+1$ and for $l=1$, $2 n_{2}=n_{1}+2$ need to be excluded, because the wave functions vanish identically for these values. Thus, the eigenvalues of the massless NB with symmetry class $l$ coincide with those of the QB with symmetry class $\tilde{l}=l+1$, where $\tilde{l}=3$ corresponds to $\tilde{l}=0$. Accordingly, the wave functions of the NB differ from those of the QB through $k_{y}$ and, in addition, in $c(k)$ by a factor $\mathrm{e}^{\mathrm{i}\left(\theta_{k}+\frac{\pi}{2}\right)}=\mathrm{i}\left(k_{x}+\mathrm{i} k_{y}\right) / k$.

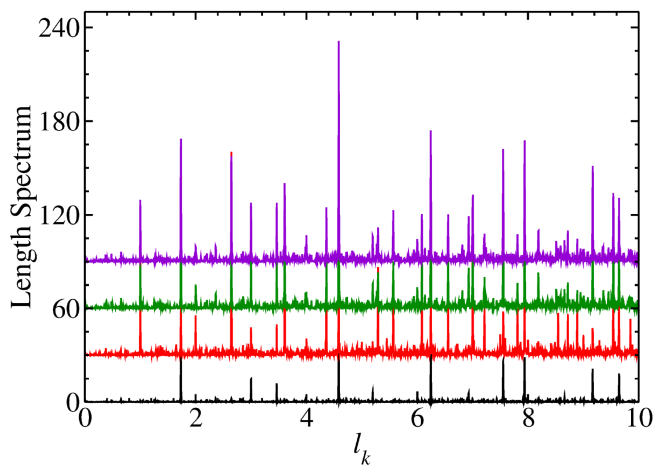

Fig. 5. Length spectra of the QB. Shown are, from bottom to top the results for the complete spectra regardless of the symmetry properties of the eigenstates (black), for the eigenstates with $l=0$ (red), with $l=1$ (dark green) and $l=2$ (violet).
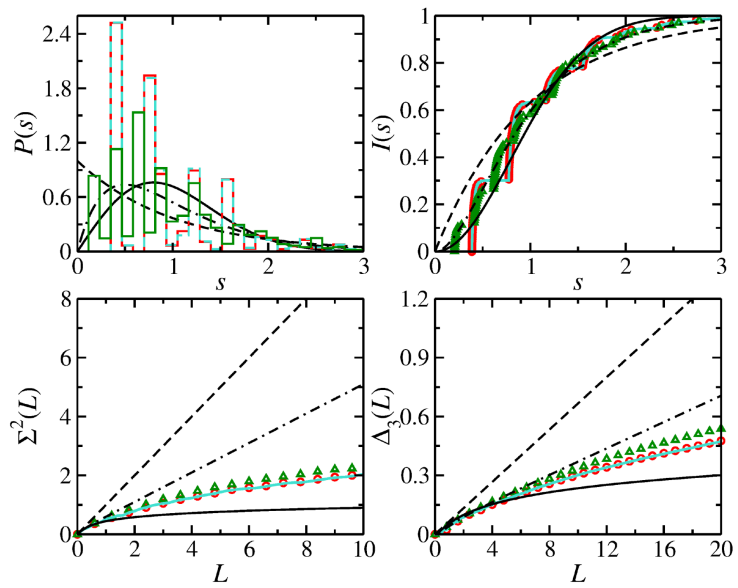

Fig. 6. Description as in Fig. 2, but for the massless neutrino billiard.

The Dirac Hamiltonian (1) does not commute with the time-reversal operator $\hat{T}$. Consequently, if the shape has no mirror symmetry the eigenvalues are non-degenerate, whereas for the ET NB the eigenvalues of the rotationally invariant states with $l=0$ coincide with those for $l=1$ except for a few values that are not possible for either of them because the corresponding first wave function component vanishes identically. This is in contrast to the nonrelativistic limit, where those corresponding to $l=1$ and $l=2$ are degenerate. This discrepancy originates from the additional spin degree of freedom leading to the entanglement of the wave-function components $\psi_{1}^{(l)}(\boldsymbol{r})$ and $\psi_{2}^{(l+1)}(\boldsymbol{r})$.

To unfold the eigenvalues we proceed as for the QB, employing Weyl's formula for NBs which coincides with the area term of that for nonrelativistic NBs, whereas it does not contain a perimeter term. Figure 6 shows the spectral properties for $l=0$ (red), $l=1$ (turquoise) and $l=2$ (dark green). The curves for $l=0$ and $l=1$ lie on top of each 


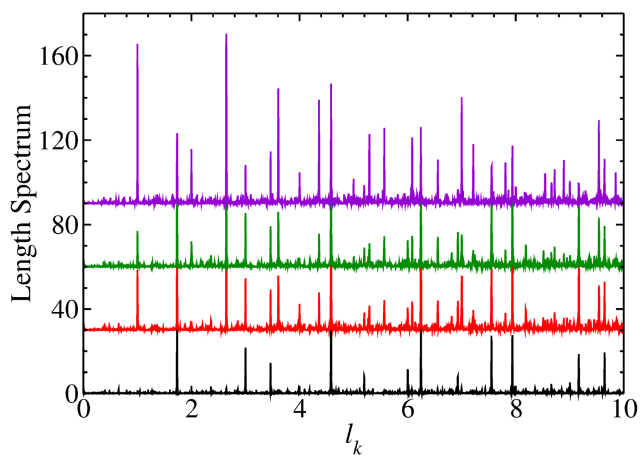

Fig. 7. Description as in Fig. 5, but for the massless neutrino billiard.

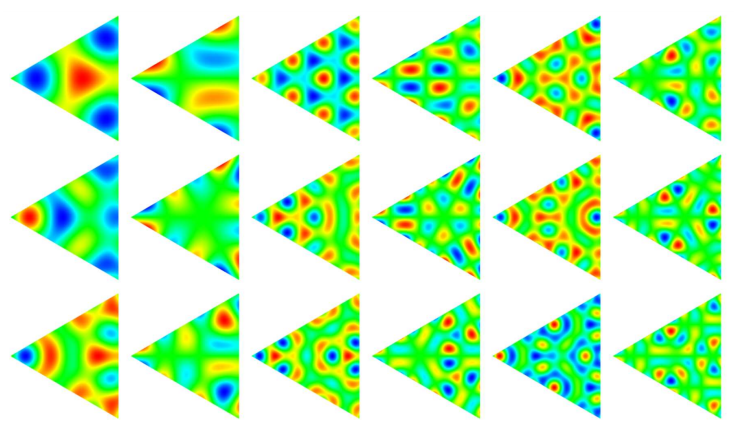

Fig. 8. Three examples of wave functions for the eigenstates with $l=0$ (top),$l=1$ (middle) and $l=2$ (bottom). Shown are the real parts of the first wave-function component (first, third, and fifth column) and the second wave-function component (second, fourth, and sixth column). The color code goes from dark blue at their smallest value to red at their maximal value.

other and are similar to those for the QB for $l=1,2$ shown in Fig. 2, whereas those for $l=2$ resemble those for the case $l=0$ in the QB. This similarity can also be observed for the ratio distributions and cumulative ratio distributions shown in the right parts of Figs. 3 and 4.

The complete and symmetry-projected length spectra are shown in Fig. 7. Those for $l=0$ (red) and $l=1$ (dark green) are identical except for a few peaks corresponding to pseudo orbits that are not present in either of the fundamental domains. In [42] symmetry-projected trace formulae were derived for systems with a discrete rotational symmetry, which interpolate between the ultrarelativistic and nonrelativistic limits and comprise pseudo orbits that exist in the fundamental domains, but not in the full system. In the ultrarelativistic limit contributions of pseudo orbits with an odd number of reflections at the boundaries of the fundamental domain cancel each other and corresponding peaks are not present in the symmetry-projected length spectra.

In Figs. 8 and 9 the real and imaginary parts of the first component (first, third, and fifth column) and associated second component (second, fourth,

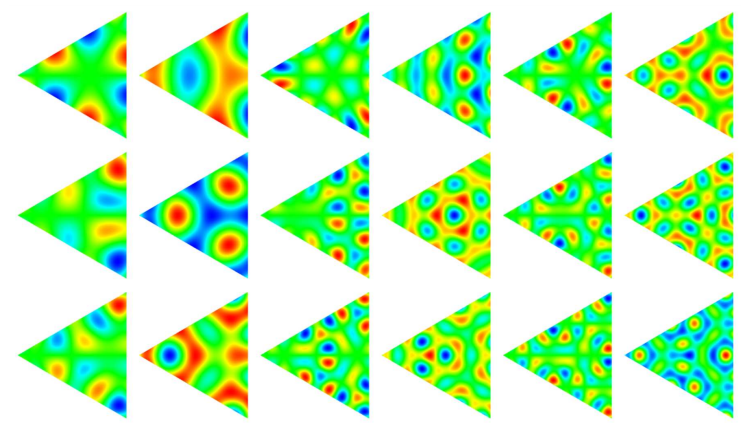

Fig. 9. Description as in Fig. 8, but for the imaginary parts.
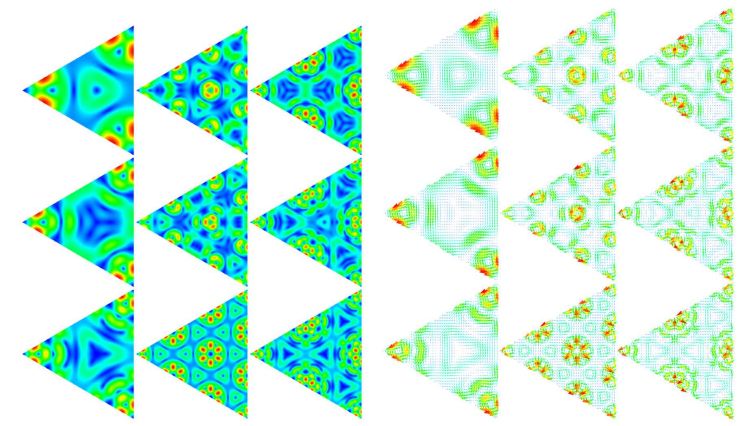

Fig. 10. Modulus (a) and direction of flow (b) of the current corresponding to the wave functions shown in Figs. 8 and 9. The color code goes from dark blue for zero intensity to red at the maximal intensity.

and sixth column) of three exemplary wave functions are shown. For $l=0$ the first wave function component is invariant under rotation by $\frac{2 \pi}{3}$, whereas the real part of the second component is antisymmetric and the imaginary part is symmetric with respect to one symmetry axis of the ET, or vice versa. For $l=1$ this rotational invariance is observed for the second component, whereas the real part of the first component is symmetric and the imaginary part antisymmetric with respect to a symmetry axis of the ET, or vice versa. For $l=2$ the real and imaginary parts of the first component are symmetric, respectively, antisymmetric, and for the second one antisymmetric, respectively, symmetric or vice versa [41, 63], i.e., $\psi_{1}(x, y)= \pm \psi_{1}^{*}(x,-y)$ and $\psi_{2}(x, y)=\mp \psi_{2}^{*}(x,-y)$, if the symmetry axis is chosen along the $x$-axis as in Figs. 8 and 9.

Figure 10 presents the modulus and direction of flow of the local current, which is defined as the expectation value of the current operator $\hat{\boldsymbol{u}}=$ $\nabla_{\boldsymbol{p}} \hat{H}_{\mathrm{D}}=c \hat{\boldsymbol{\sigma}}, \boldsymbol{u}(\boldsymbol{r})=c \boldsymbol{\psi}^{\dagger} \hat{\boldsymbol{\sigma}} \boldsymbol{\psi}$. The local current can be used to test whether the BCs are fullfilled, i.e., whether the flow of the current normal to the boundary $\boldsymbol{n}(s) \cdot \boldsymbol{u}(s)$ vanishes along the boundary, which indeed is the case. 


\section{The transition region from the ultrarelativistic to the nonrelativistic limit}

For finite mass the eigenvalues were obtained by solving the transcendental (48) and (49) numerically, and unfolded as for the nonrelativistic case, where the smooth part of the integrated spectral density is determined by fitting a second-order polynomial to it. We checked the correctness of the results, also for the massless case, by comparing with the eigenvalues obtained by solving the boundaryintegral equation derived for massive $\mathrm{NBs}$ in [42]. The spectral properties are shown in Figs. 11-16 for various values of $\tilde{\beta}$. For small $(\tilde{\beta} \lesssim 10)$ and large values $(\tilde{\beta} \gtrsim 5000)$ (left parts) they are similar to those for the QB and massless NB, respectively, i.e., nongeneric for the short-range correlations, and intermediate for the long range correlations, whereas for values of $50 \lesssim \tilde{\beta} \lesssim 1000$ the statistic is intermediate between Poisson and GOE (right parts). For $\tilde{\beta}=0$ and $l=2 k_{y}$ is an integer multiple of $\pi$ and (48) and (49) have solutions for $\pm k_{x}$ and $\pm k_{y}$. The same holds for $\tilde{\beta} \rightarrow \infty$ and $l=0$, where the corresponding subspectrum can be separated into one for which the wave functions are symmetric, and one for which they are antisymmetric with respect to all symmetry axes of the ET. This is no longer the case for finite values of $\tilde{\beta}$, i.e., the associated spectra are increasingly interspersed when decreasing $\tilde{\beta}$ from its maximal considered value $\tilde{\beta}=10000$, also for $l=2$

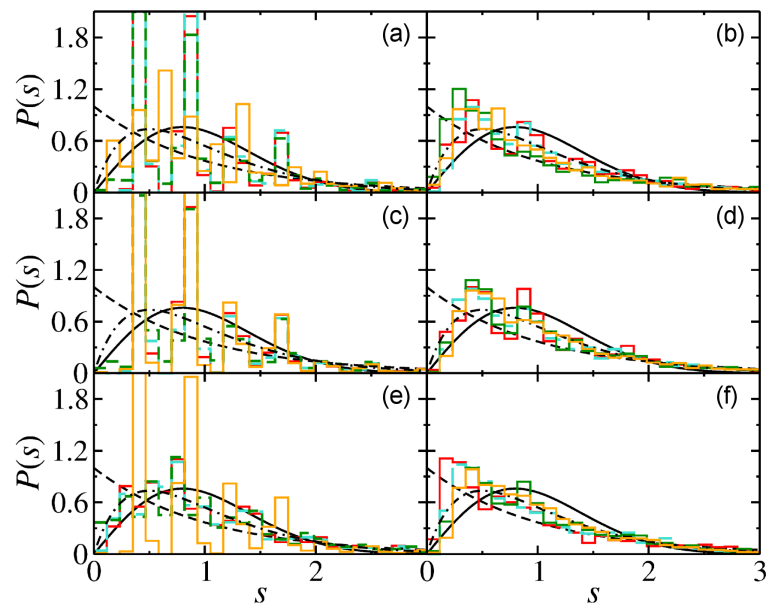

Fig. 11. Nearest neighbor spacing distribution of the massive NB. Shown are the results for the states with $l=0(\mathrm{a}, \mathrm{b}), l=1(\mathrm{c}, \mathrm{d})$ and $l=2(\mathrm{e}, \mathrm{f})$. Left: results for the massive NB with mass $\tilde{\beta}=0.1$ (red), $\tilde{\beta}=1$ (turquoise), $\tilde{\beta}=10$ (dark green) and $\tilde{\beta}=10000$ (orange). Right: results for the massive NB with mass $\tilde{\beta}=50$ (red), $\tilde{\beta}=100$ (turquoise), $\tilde{\beta}=500$ (dark green) and $\tilde{\beta}=1000$ (orange). They are compared to the spectral properties of the GOE (black full lines), Poissonian random numbers (dashed black lines) and semi-Poisson (dash-dotted black lines).

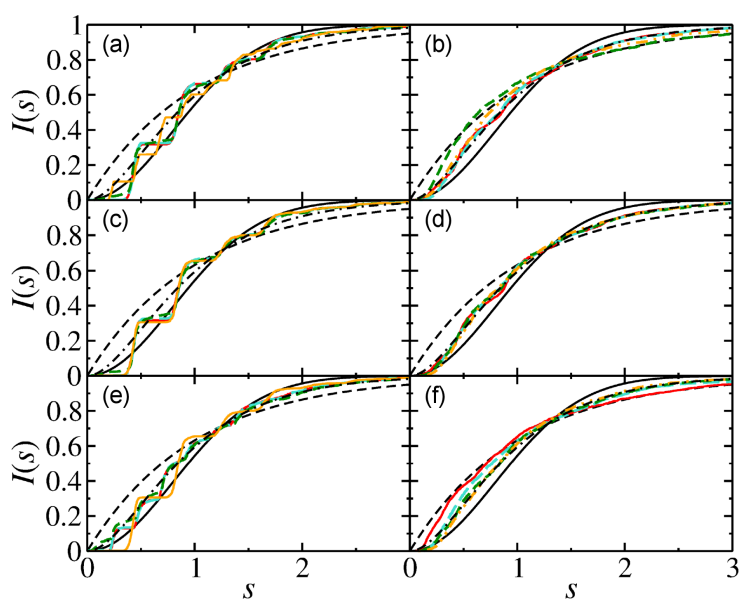

Fig. 12. Description as in Fig. 11, but for the cumulative nearest-neighbor spacing distribution.

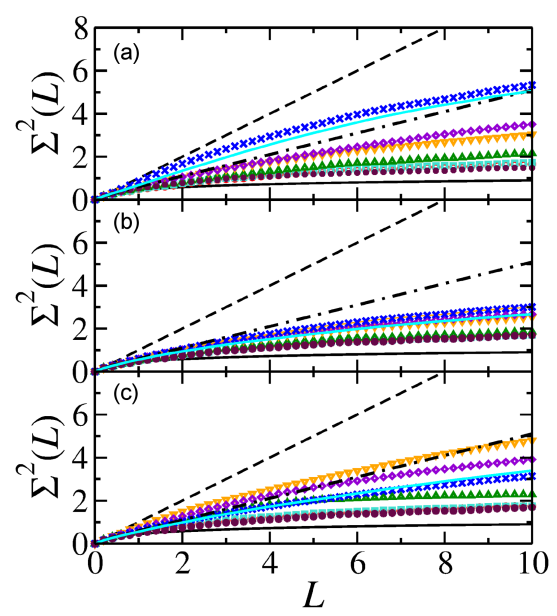

Fig. 13. Number variance of the massive NB for the states with $l=0$ (a), $l=1$ (b) and $l=2$ (c). Shown are the results for the massive NB with mass $\tilde{\beta}=0.1$ (red circles), $\tilde{\beta}=1$ (turquoise squares), $\tilde{\beta}=$ 10 (dark green upward triangles), $\tilde{\beta}=50$ (orange downward triangles), $\tilde{\beta}=100$ (violet diamonds), $\tilde{\beta}=500$ (cyan lines), $\tilde{\beta}=1000$ (blue crosses) and $\tilde{\beta}=10000$ (maroon starts). They are compared to the spectral properties of the GOE (black full lines), Poissonian random numbers (dashed black lines) and semi-Poisson (dash-dotted black lines).

when starting from $\tilde{\beta}=0$ and increasing mass sufficiently. This explains, why for $l=0$ the spectral properties are closest to Poisson for $\tilde{\beta}=1000$, and for $l=2$ for $\tilde{\beta}=50$. Generally, in the intermediatemass region the spectral properties agree well with semi-Poisson statistic.

Figure 17 shows the symmetry-projected length spectra for $l=0$ (top), $l=1$ (middle) and $l=2$ (bottom) for, from bottom to top, the massless $\mathrm{NB}, \tilde{\beta}=0.1,1,10,50,100,500,1000,10000$ and for the QB. Some of the peaks present in the length spectrum of the $\mathrm{QB}$ are missing in that of the 


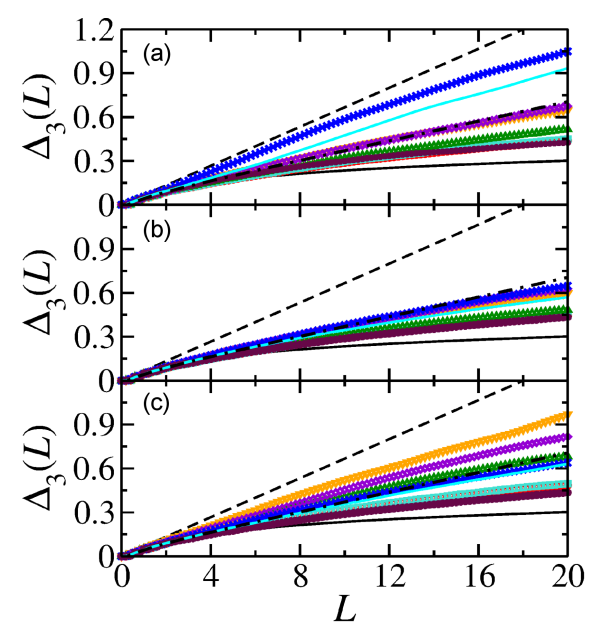

Fig. 14. Description as in Fig. 13, but for the spectral rigidity.

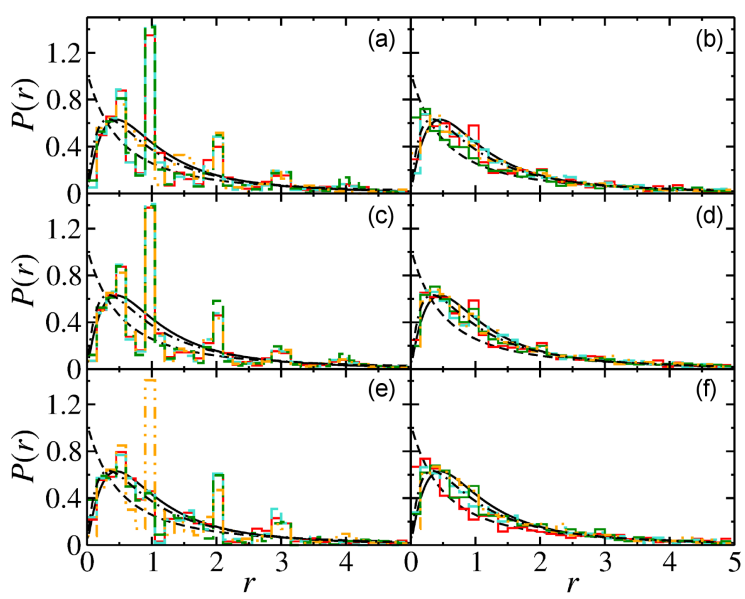

Fig. 15. Description as in Fig. 11, but for the ratio distribution.

massless NB, i.e., correspond to periodic orbits or pseudo orbits with an odd number of reflections at the boundary of the billiard or fundamental domain. These peaks already appear for small nonzero $\tilde{\beta}$. With increasing mass, NBs undergo a transition from the ultrarelativistic to the nonrelativistic limit $[42-44,61]$ and the peak heights may vary, as expected from the trace formula for massive NBs which was derived based on boundaryintegral equations in $[42,64]$. It is given in terms of a sum over periodic orbits of the CB of corresponding shape and contains an extra phase factor as compared to the trace formula for the nonrelativistic QB which depends on $\tilde{\beta}$ and may lead in the symmetry-projected trace formula to constructive instead of destructive interference and thus to the variations in peak heights with increasing $\tilde{\beta}$. For small $\tilde{\beta}$ the length spectra for $l=0$ and $l=1$ are similar, for large $\tilde{\beta}$ those for $l=1$ and $l=2$.

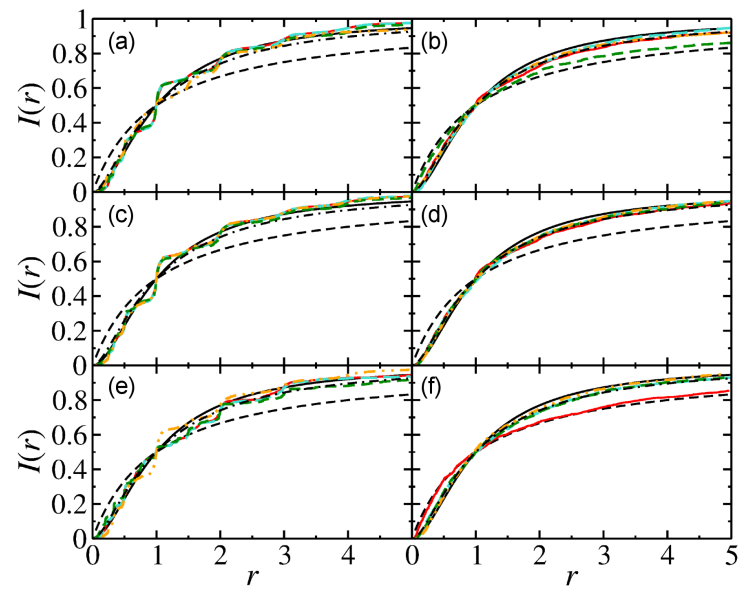

Fig. 16. Description as in Fig. 11, but for the cumulative ratio distribution.
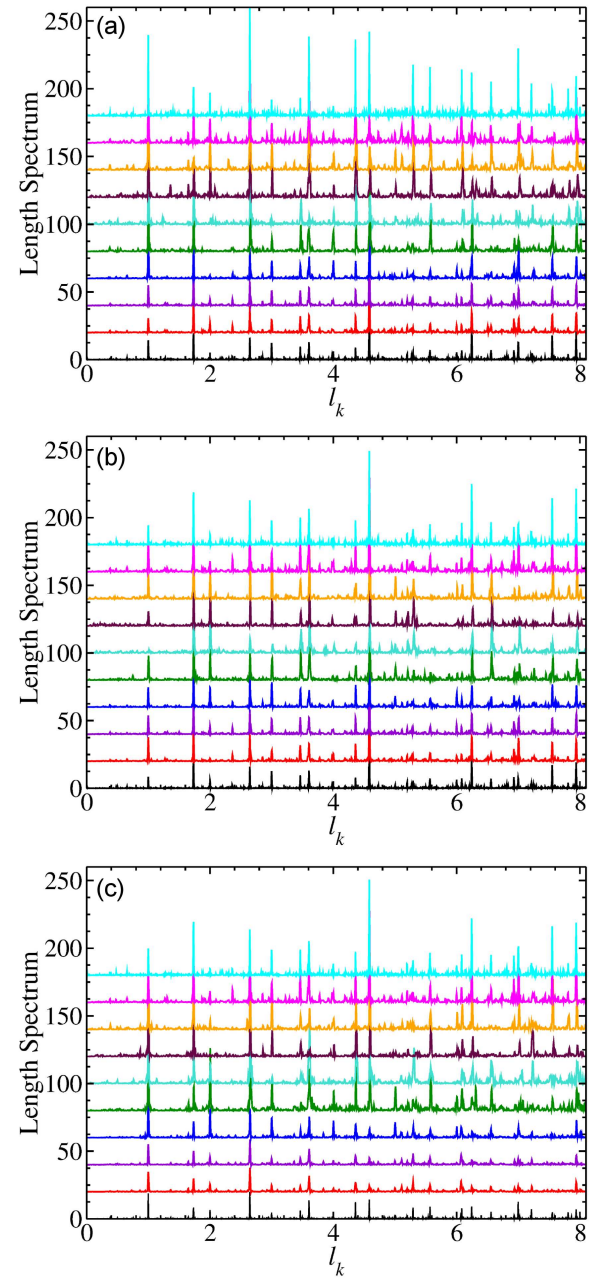

Fig. 17. Length spectra of the states with $l=0$ (a), $l=1$ (b) and $l=2$ (c) for, from bottom to top, the NBs with mass $\tilde{\beta}=0$ (black), $\tilde{\beta}=0.1$ (red), $\tilde{\beta}=1$ (violet), $\tilde{\beta}=10$ (blue), $\tilde{\beta}=50$ (dark green), $\tilde{\beta}=100$ (turquoise), $\tilde{\beta}=500$ (maroon), $\tilde{\beta}=1000$ (orange), $\tilde{\beta}=10000$ (magenta), and the QB (cyan). 


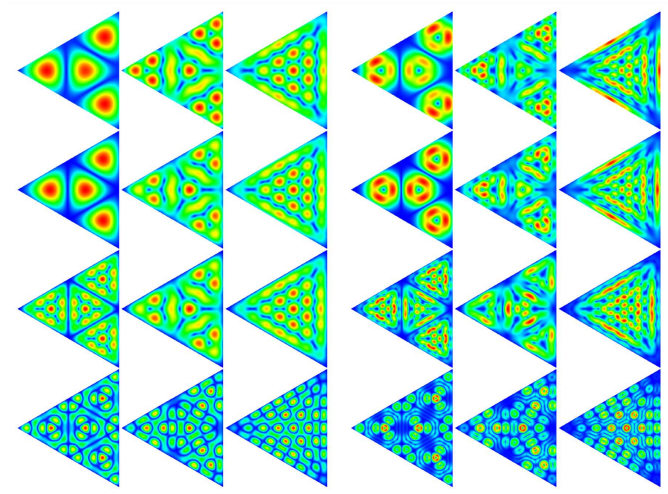

Fig. 18. Three examples of the modulus of the first wave-function component (left three columns) and the current (right three columns) for $l=0$ for, from top to bottom, $\tilde{\beta}=10,50,500,10000$.

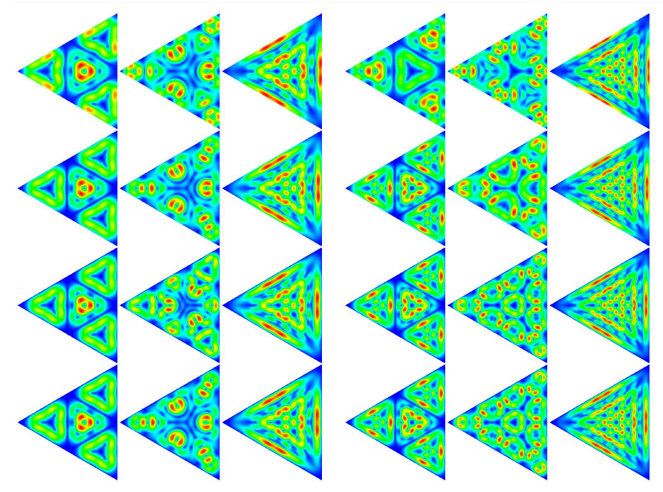

Fig. 19. Three examples of the modulus of the current for $l=1$ (left three columns) and $l=2$ (right three columns) for, from top to bottom, $\tilde{\beta}=10,50$, 500, 10000 .

Figure 18 shows three examples for the modulus of the first wave function component (left three columns) and the corresponding current (right three columns) for $l=0$ and, from top to bottom, $\tilde{\beta}=10$, $50,500,10000$. For $\tilde{\beta} \lesssim 1000$ the wave function patterns and also the patterns of the currents do not show a clear nodal domain pattern, since they are complex. With increasing $\tilde{\beta}$ the $\mathrm{BC}(2)$ approaches the Dirichlet BC, as clearly visible, and the wave function and current patterns become well discernible indicating that $\tilde{\psi}_{1}(\boldsymbol{r})$ is real for the largest value of $\tilde{\beta}$. Indeed, for $\tilde{\beta}=10000$ the eigenvalues coincide with those of the QB. Note, that with increasing $\tilde{\beta}$ the ratio $\psi_{1}(\boldsymbol{r}) / \psi_{2}(\boldsymbol{r})$ approaches zero proportional to $\mathcal{K}$, and the wave-function components $\tilde{\psi}_{1,2}(\boldsymbol{r})$ decouple.

Figure 19 shows three examples for the local currents for states with $l=1$ (left three columns) and $l=2$ (right three columns). With increasing $\tilde{\beta}$ the modulus of the local current becomes vanishingly small at the boundary of the ET, thus indicating that the wave-function components are decoupled and obey the Dirichlet BCs.

\section{Conclusions}

We present analytical results for the eigenvalues and wave functions of relativistic ET NBs and employ them to thoroughly analyse the spectral properties of NBs with threefold symmetry for the ultrarelativistic limit corresponding to mass $\tilde{\beta}=0$ and in the transition region to the nonrelativistic limit, which is reached for $\tilde{\beta} \rightarrow \infty$. For this we separate the eigenstates of the $\mathrm{QB}$ and the $\mathrm{NB}$ according to their transformation properties under rotation by $\frac{2 \pi}{3}$ into three subspectra characterized by $l=0,1,2$. Generally, the Dirac equation and $\mathrm{BCs}$ for a NB with threefold-symmetry relate the wave function components $\tilde{\psi}_{1}^{(l)}(\boldsymbol{r})$ to $\tilde{\psi}_{2}^{(l-1)}(\boldsymbol{r})$, i.e., the first and second wave-function component transform differently under rotation by $\frac{2 \pi}{3}$. In the nonrelativistic limit the eigenvalues with $l=1,2$ are degenerate, whereas in the ultrarelativistic limit those corresponding to $l=0,1$ coincide. This discrepancy is attributed to the additional spin degree of freedom. Accordingly, the fluctuation properties in the eigenvalue spectra of the ET QB corresponding to $l=1,2$ and $l=0$ coincide with those of the massless ET NB with $l=0,1$, respectively, $l=2$. In the nonrelativistic and ultrarelativistic limit the short-range correlations are non-generic, as they are not described by a random-matrix model or Poisson statistics, whereas the long-range correlations exhibit intermediate statistic and, actually, are close to semiPoisson. For $\tilde{\beta} \rightarrow \infty$ the subspectrum labeled by $l=0$ and, similarly that for $\tilde{\beta}=0$ and $l=2$ can be further separated. Yet, this is no longer possible for finite masses. For these values of $l$ we observe an approach of the spectral properties to Poisson when $\tilde{\beta}$ moves away from the corresponding limiting value, whereas for $l=1$ the spectral properties barely change with $\tilde{\beta}$.

\section{Acknowledgments}

This work was supported by the National Natural Science Foundation of China through Grants Nos. 11775100, 11961131009, and 12047501.

\section{References}

[1] Y.G. Sinai, Russ. Math. Surv. 25, 137 (1970).

[2] L.A. Bunimovich, Commun. Math. Phys. 65, 295 (1979).

[3] M.V. Berry, Eur. J. Phys. 2, 91 (1981).

[4] Chaos and Quantum Physics, Eds. M. Giannoni, A. Voros, J. Zinn-Justin, Elsevier, Amsterdam 1989.

[5] H.-J. Stöckmann, J. Stein, Phys. Rev. Lett. 64, 2215 (1990). 
[6] A. Richter, in: Emerging Applications of Number Theory, The IMA Volumes in Mathematics and its Applications, Eds. D.A. Hejhal, J. Friedman, M.C. Gutzwiller, A.M. Odlyzko, Vol. 109, Springer, New York 1999, p. 479.

[7] F. Haake, S. Gnutzmann, M. Kuś, Quantum Signatures of Chaos, Springer-Verlag, Heidelberg 2018.

[8] S. Sridhar, Phys. Rev. Lett. 67, 785 (1991).

[9] H.-D. Gräf, H.L. Harney, H. Lengeler, C.H. Lewenkopf, C. Rangacharyulu, A. Richter, P. Schardt, H.A. Weidenmúller, Phys. Rev. Lett. 69, 1296 (1992).

[10] J. Stein, H.-J. Stöckmann, Phys. Rev. Lett. 68, 2867 (1992)

[11] P. So, S.M. Anlage, E. Ott, R. Oerter, Phys. Rev. Lett. 74, 2662 (1995)

[12] S. Deus, P.M. Koch, L. Sirko, Phys. Rev. E 52, 1146 (1995).

[13] B. Dietz, A. Richter, Chaos 25, 097601 (2015).

[14] M.V. Berry, M. Tabor, J. Phys. A 10, 371 (1977).

[15] M. Berry, Structural Stability in Physics, Pergamon Press, Berlin 1979.

[16] G. Casati, F. Valz-Gris, I. Guarnieri, Lett. Nuovo Cim. 28, 279 (1980).

[17] O. Bohigas, M.J. Giannoni, C. Schmit, Phys. Rev. Lett. 52, 1 (1984).

[18] M.V. Berry, J. Phys. A 10, 2083 (1977).

[19] M.L. Mehta, Random Matrices, Academic Press, London 1990.

[20] S. Heusler, S. Müller, A. Altland, P. Braun, F. Haake, Phys. Rev. Lett. 98, 044103 (2007).

[21] M.C. Gutzwiller, J. Math. Phys. 12, 343 (1971).

[22] M.C. Gutzwiller, Chaos in Classical and Quantum Mechanics, Springer, 1990.

[23] M.V. Berry, R.J. Mondragon, Proc. R. Soc. Lond. A 412, 53 (1987).

[24] H. Weyl, Z. Phys. 56, 330 (1929).

[25] J. Bolte, S. Keppeler, Ann. Phys. 274, 125 (1999).

[26] J. Wurm, K. Richter, I. Adagideli, Phys. Rev. B 84, 075468 (2011).

[27] K.S. Novoselov, A.K. Geim, S.V. Morozov, D. Jiang, Y. Zhang, S.V. Dubonos, I.V. Grigorieva, A.A. Firsov, Science 306, 666 (2004).

[28] C.W.J. Beenakker, Rev. Mod. Phys. 80, 1337 (2008).

[29] A.H. Castro Neto, F. Guinea, N.M.R. Peres, K.S. Novoselov, A.K. Geim, Rev. Mod. Phys. 81, 109 (2009).
[30] P.G. Silvestrov, K.B. Efetov, Phys. Rev. Lett. 98, 016802 (2007).

[31] L.A. Ponomarenko, F. Schedin, M.I. Katsnelson, R. Yang, E.W. Hill, K.S. Novoselov, A.K. Geim, Science 320, 5874 (2008).

[32] F. Libisch, C. Stampfer, J. Burgdörfer, Phys. Rev. B 79, 115423 (2009).

[33] J. Wurm, A. Rycerz, I. Adagideli, M. Wimmer, K. Richter, H.U. Baranger, Phys. Rev. Lett. 102, 056806 (2009).

[34] L. Huang, Y.-C. Lai, D.K. Ferry, S.M. Goodnick, R. Akis, Phys. Rev. Lett. 103, 054101 (2009).

[35] A. Rycerz, Phys. Rev. B 85, 245424 (2012).

[36] A. Rycerz, Phys. Rev. B 87, 195431 (2013).

[37] M. Polini, F. Guinea, M. Lewenstein, H.C. Manoharan, V. Pellegrini, Nat. Nanotechnol. 8, 625 (2013).

[38] B. Dietz, T. Klaus, M. Miski-Oglu, A. Richter, Phys. Rev. B 91, 035411 (2015).

[39] B. Dietz, T. Klaus, M. Miski-Oglu, A. Richter, M. Wunderle, C. Bouazza, Phys. Rev. Lett. 116, 023901 (2016).

[40] L. Huang, H.-Y. Xu, C. Grebogi, Y.-C. Lai, Phys. Rep. 753, 1 (2018).

[41] B. Dietz, A. Richter, Phys. Scr. 94, 014002 (2019).

[42] B. Dietz, Z.-Y. Li, Phys. Rev. E 102 , 042214 (2020).

[43] G. Baym, Lectures on Quantum Mechanics, CRC Press, Boca Raton (FL) 2018.

[44] M.-Y. Song, Z.-Y. Li, H.-Y. Xu, L. Huang, Y.-C. Lai, Phys. Rev. Res. 1, 033008 (2019).

[45] J.M. Robbins, Phys. Rev. A 40, 2128 (1989).

[46] S.C. Creagh, R.G. Littlejohn, Phys. Rev. A 44, 836 (1991).

[47] S.C. Creagh, R.G. Littlejohn, J. Phys. A 25, 1643 (1992).

[48] T.H. Seligman, H.A. Weidenmüller, J. Phys. A 27, 7915 (1994).

[49] F. Leyvraz, C. Schmit, T.H. Seligman, J. Phys. A 29, L575 (1996).

[50] J.P. Keating, J.M. Robbins, J. Phys. A 30, L177 (1996).

[51] P. Braun, F. Leyvraz, T.H. Seligman, New J. Phys. 13, 063027 (2011).

[52] C.H. Joyner, S. Müller, M. Sieber, J. Phys. A 45, 205102 (2012).

[53] P.J. Richens, M.V. Berry, Physica D 2, 495 (1981).

[54] H.R. Krishnamurthy, H.S. Mani, H.C. Verma, J. Phys. A 15, 2131 (1982). 
[55] P. Bellomo, Pramana J. Phys. 44, 85 (1995).

[56] I.O. Sukharevsky, M. Lebental, B. Dietz, C. Lafargue, S. Bittner, in: Laser Resonators, Microresonators, and Beam Control XX, Eds. A.V. Kudryashov, A.H. Paxton, V.S. Ilchenko, Vol. 10518, International Society for Optics and Photonics (SPIE), 2018, p. 306.

[57] I.O. Sukharevsky, M. Lebental, B. Dietz, C. Lafargue, S. Bittner, Opt. Eng. 58, 1 (2019).

[58] P. Potasz, A.D. Güçlü, P. Hawrylak, Phys. Rev. B 81, 033403 (2010).

[59] A.V. Rozhkov, F. Nori, Phys. Rev. B 81, 155401 (2010).

[60] M. Zarenia, A. Chaves, G.A. Farias, F.M. Peeters, Phys. Rev. B 84, 245403 (2011).

[61] W.A. Gaddah, J. Phys. A 51, 385304 (2018).

[62] P. Yu, B. Dietz, L. Huang, New J. Phys. 21, 073039 (2019).

[63] P. Yu, B. Dietz, H.-Y. Xu, L. Ying, L. Huang, Y.-C. Lai, Phys. Rev. E 101, 032215 (2020).
[64] W. Zhang, B. Dietz, Phys. Rev. B 104 064310 (2021).

[65] J.P. Keating, J.M. Robbins, J. Phys. A 30, L177 (1997).

[66] C. Dembowski, H.-D. Gräf, A. Heine, H. Rehfeld, A. Richter, C. Schmit, Phys. Rev. E 62, R4516 (2000).

[67] Leçons sur la Théorie Mathématique d'Elasticité des Cores Solides, Ed. M.G. Lamé, Bachelier, Paris 1852 (in French).

[68] P. McIsaac, IEEE Trans. Microwave Theory Techn. 23, 421 (1975).

[69] E.B. Bogomolny, U. Gerland, C. Schmit, Phys. Rev. E 59, R1315 (1999).

[70] Y.Y. Atas, E. Bogomolny, O. Giraud, G. Roux, Phys. Rev. Lett. 110, 084101 (2013).

[71] N. Rosenzweig, C. Porter, Phys. Rev. 120, 1698 (1960).

[72] V. Oganesyan, D.A. Huse, Phys. Rev. B 75, 155111 (2007).

[73] E. Bogomolny, U. Gerland, C. Schmit, Eur. Phys. J. B 19, 121 (2001). 